\title{
Random Complex Zeroes and Random Nodal Lines
}

\author{
Fedor Nazarov and Mikhail Sodin *
}

To The MEMory OF Oded SChramm

\begin{abstract}
.
In these notes, we describe the recent progress in understanding the zero sets of two remarkable Gaussian random functions: the Gaussian entire function with invariant distribution of zeroes with respect to isometries of the complex plane, and Gaussian spherical harmonics on the two-dimensional sphere.

These notes consist of two almost independent parts. In both of them, we talk about zeroes of special Gaussian random functions. To understand them, we had to combine various tools from complex and real analysis with rudimentary probabilistic methods. We think that the results and techniques presented here can serve as guidelines in other problems of similar nature arising in analysis, mathematical physics, and probability theory.

The function $F$ that we consider in the first part is a random analytic function of one complex variable. In this case, one can recover the zeroes of $F$ by applying the Laplacian to $\log |F|$. This paves the way for using complex analysis tools, and for this reason, the problems that we discuss in the first part are pretty well understood by now, though some intriguing questions still remain open.

In the second part, we deal with topological properties of the zero sets of random (real-valued) functions of several real variables. This is an area with wealth of interesting and difficult questions and with very few advances. In essence, in this part, the reader will find a discussion of one recent theorem on the number of connected components of zero sets of Gaussian spherical harmonics along with various open questions.
\end{abstract}

\section{PART I. RANDOM COMPLEX ZEROES}

The study of zeroes of random polynomials and random analytic functions has a long history. It started with the pioneering works of Kac, Littlewood, Offord, Rice, and Wiener, and was later continued by Hammersley, Kahane, Maslova, and many others. The subject was revived in the 1990's by several groups of researchers (Bogomolny-Bohigas-Leboeuf, Shub-Smale, Edelman-Kostlan, Ibragimov-Zeitouni,

\footnotetext{
*This work is partially supported by grant No. 2006136 of the United States - Israel Binational Science Foundation.
} 
Hannay, Bleher-Shiffman-Zelditch, Nonnenmacher-Voros) who came from very different areas and established new links to mathematical physics, probability theory, and complex geometry. Some of these results were surveyed in the lectures by Zelditch [54 and Sodin [44; see also an introductory article 33] and the recent book by Hough, Krishnapur, Peres, and Virág [21.

In particular, Kostlan, Bogomolny-Bohigas-Leboeuf, Shub-Smale, and Hannay introduced a remarkable construction of random Gaussian entire functions with translation invariant distribution of their zeroes. Let

$$
F(z)=\sum_{n \geq 0} \zeta_{n} \frac{z^{n}}{\sqrt{n !}}
$$

where $\zeta_{n}$ are independent standard complex Gaussian random coefficients (i.e., the density of $\zeta_{k}$ with respect to the Lebesgue measure in $\mathbb{C}$ is $\frac{1}{\pi} e^{-|\zeta|^{2}}$ ). The distribution of the random function $F$ is invariant with respect to rotations around the origin, but it is not translation invariant, for instance, because $\mathcal{E}|F(z)|^{2}=$ $e^{|z|^{2}}$ (here and below, $\mathcal{E}$ means the mathematical expectation). However, the distribution of the zero set $\mathcal{Z}=F^{-1}\{0\}$ is translation invariant. One of the ways to see this is to check that the Gaussian random function

$$
F_{\lambda}(z)=F(z+\lambda) e^{-z \bar{\lambda}-\frac{1}{2}|\lambda|^{2}}, \quad \lambda \in \mathbb{C},
$$

has the same covariance function as $F$ :

$$
\mathcal{E}\{F(z) \overline{F(w)}\}=\mathcal{E}\left\{F_{\lambda}(z) \overline{F_{\lambda}(w)}\right\}=e^{z \bar{w}}
$$

which is nothing else but the reproducing kernel in the classical Fock-Bargmann space of entire functions. This coincidence is not accidental 33. Moreover, due to remarkable Calabi's rigidity [21, Section 2.5], this is the only translation invariant zero set of a Gaussian entire function up to scaling. We call the function $F$ the Gaussian Entire Function (G.E.F., for short).

It is worth mentioning that there exist similar constructions for other domains with transitive groups of isometries (the hyperbolic plane, the Riemann sphere, the cylinder and the torus).

\section{Linear statistics}

One of the most traditional ways to study asymptotic properties of a random point process $Z$ in the plane is to take a test-function $h$, and to look at the asymptotic behaviour of the linear statistics

$$
n_{Z}(r, h)=\sum_{a \in Z} h\left(\frac{a}{r}\right)
$$

as $r \rightarrow \infty$. We put $n(r, h)=n_{\mathcal{Z}}(r, h)$. An easy computation shows that

$$
\mathcal{E} n(r, h)=\frac{r^{2}}{\pi} \int_{\mathbb{R}^{2}} h .
$$


If $h=\mathbb{1}_{E}$ is the indicator function of a set $E$, then $n\left(r, \mathbb{1}_{E}\right)=n(r E)$ is the number of zeroes in the set $r E$.

A usual "triad" in the study of linear statistics is

$$
\text { VARIANCE, ASYMPTOTIC NORMALITY, LARGE FLUCTUATIONS }
$$

First, we'll discuss the variance, which is the easiest part of the triad.

\subsection{The variance.}

Theorem 1.1 (The variance). For every non-zero function $h \in\left(L^{1} \cap L^{2}\right)\left(\mathbb{R}^{2}\right)$ and every $R>0$,

$$
\operatorname{Var} n(r, h)=r^{2} \int_{\mathbb{R}^{2}}|\widehat{h}(\lambda)|^{2} M\left(r^{-1} \lambda\right) \mathrm{d} m(\lambda)
$$

where

$$
M(\lambda)=\pi^{3}|\lambda|^{4} \sum_{\alpha \geq 1} \frac{1}{\alpha^{3}} e^{-\frac{\pi^{2}}{\alpha}|\lambda|^{2}}
$$

and

$$
\widehat{h}(\lambda)=\int_{\mathbb{R}^{2}} h(x) e^{-2 \pi \mathrm{i}\langle\lambda, x\rangle} \mathrm{d} m(x)
$$

is the Fourier transform of $h$.

This theorem was proven in 34. The asymptotic of the variance had been known for two special cases since the work by Forrester and Honner [17]: if $h \in C_{0}^{2}$ (i.e., $h$ is a $C^{2}$-function with compact support), then

$$
\operatorname{Var} n(r, h)=\frac{\zeta(3)+o(1)}{16 \pi r^{2}}\|\Delta h\|_{L^{2}}^{2}, \quad r \rightarrow \infty,
$$

while for bounded domains $G$ with piecewise smooth boundary,

$$
\operatorname{Var} n(r G)=\frac{\zeta(3 / 2)+o(1)}{8 \pi^{3 / 2}} r L(\partial G), \quad r \rightarrow \infty .
$$

Here, $\zeta(\cdot)$ is Riemann's zeta-function.

A less precise form of Theorem 1.1 might be more illustrative:

$$
\operatorname{Var} n(r, h) \simeq r^{-2} \int_{|\lambda| \leq r}|\widehat{h}(\lambda)|^{2}|\lambda|^{4} \mathrm{~d} m(\lambda)+r^{2} \int_{|\lambda| \geq r}|\widehat{h}(\lambda)|^{2} \mathrm{~d} m(\lambda),
$$

where the notation $A \simeq B$ means that the quotient $B / A$ is bounded from below and from above by positive numerical constants. The right-hand side of (1.3) interpolates $\|h\|_{L^{2}(m)}^{2}$ and $\|\Delta h\|_{L^{2}(m)}^{2}$. 


\subsection{Digression: "superhomogeneous" point processes.}

By (1.2), the random zero process $\mathcal{Z}$ belongs to the family of so called superhomogeneous translation invariant point processes with fluctuations of the number of points in large domains proportional to the length of the boundary rather than to the area, as it would be, say, for the Poisson process.

A "toy model" for such processes is the point process

$$
\mathcal{S}=\left\{\omega+\zeta_{\omega}: \omega \in \sqrt{\pi} \mathbb{Z}^{2}\right\}
$$

obtained by perturbing the lattice $\sqrt{\pi} \mathbb{Z}^{2}$ by independent standard complex Gaussian random variables $\zeta_{\omega}$. The normalization by $\sqrt{\pi}$ is not essential here, it is introduced to have asymptotically the same mean number of points in large areas as our process $\mathcal{Z}$ has. The choice of the square lattice is not essential either.

Curiously, the same kernel $e^{z \bar{w}}$ that occurs in the definition of random complex zeroes generates by a very different construction another interesting superhomogeneous point process $\mathcal{G}$, namely, the determinantal process whose $k$-point functions can be expresed in terms of the determinants formed by this kernel.

$$
\rho\left(z_{1}, \ldots, z_{k}\right)=\pi^{-k} e^{-\sum_{i=1}^{k}\left|z_{i}\right|^{2}} \operatorname{det}\left\|e^{z_{i} \overline{z_{j}}}\right\|_{1 \leq i, j \leq k} .
$$

This process arises as the large $N$ limit of eigenvalues of Ginibre ensemble of $N \times N$ matrices with independent standard complex Gaussian entries, and we will call it the limiting Ginibre process. It is known that the Ginibre point process is
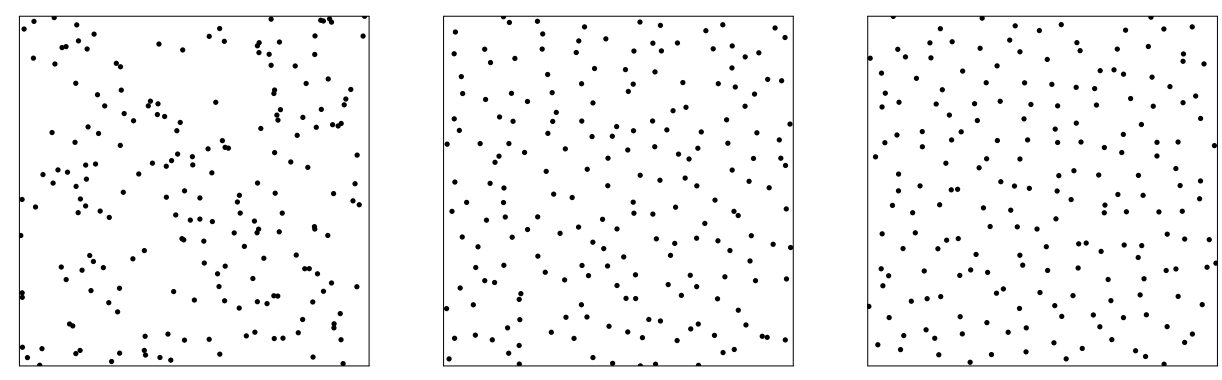

Figure 1. Samples of the Poisson process (figure by B. Virág), limiting Ginibre process, and zeroes of a GEF (figures by M. Krishnapur). Some properties of the last two processes are quite different, though the eye does not easily distinguish between them.

a special, explicitly solvable case of a one-component plasma of charged particles of one sign confined by a uniform background of the opposite sign. Though the one-component plasma has been studied by physicists for a long time, it seems that most of rigorous results still pertain only to the very special case of the Ginibre ensemble.

Resemblances and differences between the processes $\mathcal{Z}, \mathcal{S}$, and $\mathcal{G}$ were discussed both in the physical and the mathematical literature. For instance, the behaviour of smooth linear statistics for these three processes is quite different. In particular, decay of the variance of smooth linear statistics (1.1) distinguishes the zero process 
$\mathcal{Z}$ from the processes $\mathcal{G}$ and $\mathcal{S}$, since for the latter two processes, the variance of smooth linear statistics tends to the positive limit proportional to $\|\nabla h\|_{L^{2}(m)}^{2}$.

\subsection{Asymptotic normality of fluctuations.}

1.3.1. Normal fluctuations. We say that the linear statistics $n(r, h)$ have asymptotically normal fluctuations if the normalized linear statistics

$$
\frac{n(r, h)-\mathcal{E} n(r, h)}{\sqrt{\operatorname{Var} n(r, h)}}
$$

converge in distribution to the standard (real) Gaussian random variable as $r \rightarrow \infty$.

Let $C_{0}^{\alpha}, \alpha>0$, be the class of compactly supported $C^{\alpha}$-functions, by $C_{0}^{0}$ we denote the class of bounded compactly supported measurable functions.

Theorem 1.2 (Asymptotic normality). Suppose that $h \in C_{0}^{\alpha}$ with some $\alpha \geq 0$, and that for some $\varepsilon>0$ and for every sufficiently big $r$, we have

$$
\operatorname{Var} n(r, h)>r^{-2 \alpha+\varepsilon} .
$$

Then the linear statistics $n(r, h)$ have asymptotically normal fluctuations.

Note that by (1.3), we always have $\operatorname{Var} n(r, h) \geq c(h) r^{-2}$ with positive $c(h)$ independent of $r$. Hence, for $\alpha>1$, condition (1.5) holds automatically, and we obtain the following

Corollary 1.1. Suppose that $h \in C_{0}^{\alpha}$ with $\alpha>1$. Then the linear statistics $n(r, h)$ have asymptotically normal fluctuations.

Using estimate (1.3), one can show that for any bounded measurable set $E$ of positive area, $\operatorname{Var} n(r E) \gtrsim r$, cf. (1.2). Hence,

Corollary 1.2. Let $E$ be a bounded measurable set of positive area. Then the number of random complex zeroes $n(r E)$ on the set $r E$ has asymptotically normal fluctuations.

1.3.2. Abnormal fluctuations of linear statistics. Do there exist $C_{0}^{\alpha}$-functions $h$ with abnormal asymptotic behaviour of linear statistics $n(r, h)$ ? The answer is "yes", and the simplest example is provided by the function $h_{\alpha}=|x|^{\alpha} \psi(x)$, where $\psi$ is a smooth cut-off that equals 1 in a neighborhood of the origin. Clearly, $h_{\alpha} \in C_{0}^{\alpha}$ and it is not difficult to show that $\operatorname{Var} n\left(r, h_{\alpha}\right) \simeq r^{-2 \alpha}$. This shows that Theorem 1.2 is sharp on a rough power scale. The reason for the loss of asymptotic normality is that only a small neighbourhood of the origin where $h_{\alpha}$ loses its smoothness contributes to the variance of $n\left(r, h_{\alpha}\right)$. This neighbourhood contains a bounded number of zeroes of $F$, which is not consistent with the idea of normal fluctuations. 
1.3.3. Comments and questions. Theorem 1.2 was preceded by a result of Sodin and Tsirelson [45, Part I]. Using the moment method and the diagrams, they showed that the fluctuations are asymptotically normal provided that $h \in C_{0}^{2}$. Their technique works in several other cases, for instance, when $h=\mathbb{1}_{G}$ is the indicator function of a bounded domain $G$ with a piecewise smooth boundary. However, it seems very difficult to adapt it for proving Theorem 1.2 in its full generality.

In the case $\alpha>0$, the proof of Theorem 1.2 uses a classical idea of S.Bernstein to approximate the random variable $n(R, h)$ by a sum of a large number of independent random variables with negligible error. Such approximation becomes possible only after we separate the high and the low frequencies in $h$. In this approach, independence appears as a consequence of the almost independence of the values of the G.E.F. at large distances, which we'll discuss below in Section 3.1 We do not know whether asymptotic normality holds for all functions $h \in C_{0}^{1}$, or whether the condition $r^{2 \alpha} \operatorname{Var} n(r, h) \rightarrow \infty$ is already sufficient for asymptotic normality of linear statistics associated with a $C_{0}^{\alpha}$-function. Also, we believe that the assertion of Theorem 1.2 can be extended to functions $h \in C^{\alpha} \cap L_{0}^{2}$ with $-1<\alpha<0$ but our current techniques seem insufficient to handle this case properly.

In the case $\alpha=0$, the proof uses a different idea which comes from statistical mechanics. First, we show that $k$-point functions of the zero process $\mathcal{Z}$ are clustering, see Section 3.2 for the precise statement. Then, using clustering, we estimate the cumulants of the random variable $n(r, h)$.

It is interesting to juxtapose Theorem 1.2 with what is known for the limiting Ginibre process $\mathcal{G}$ described above. For bounded compactly supported functions $h$, a counterpart of Theorem 1.2 is a theorem of Soshnikov. In 47, he proved among other things that for arbitrary determinantal point processes, the fluctuations of linear statistics associated with a compactly supported bounded positive function are normal if the variance grows at least as a positive power of expectation as the intensity tends to infinity. A counterpart of the limiting case $\alpha=2$ in Theorem 1.2 (that is, of the result from [45. Part I]) was recently found by Rider and Virág in [42. They proved that the fluctuations for linear statistics of process $\mathcal{G}$ are normal when the test function $h$ belongs to the Sobolev space $W_{1}^{2}$. It is not clear whether there is any meaningful statement interpolating between the theorems of Soshnikov and Rider and Virág. It can happen that our Theorem 1.2 simply has no interesting counterpart for the process $\mathcal{G}$. It is also worth mentioning that the proofs in the determinantal case are quite different from ours. They are based on peculiar combinatorial identities for the cumulants of linear statistics that are a special feature of determinantal point processes.

\subsection{Probability of large fluctuations.}

Now, we turn to the probability of exponentially rare events that, for some $r \gg 1$, $|n(r, h)-\mathcal{E} n(r, h)|$ is much bigger than $\sqrt{\operatorname{Var}(n(r, h)}$. Mostly, we consider the case when $h$ is the indicator function of the unit disk $\mathbb{D}$; i.e., we deal with the number $n(r)$ of random zero points in the disk of large radius $r$ centered at the origin. Recall that $\mathcal{E} n(r)=r^{2}$ and $\mathcal{E}\left\{\left(n(r)-r^{2}\right)^{2}\right\} \sim c r$ for $r \rightarrow \infty$ (with some 
$c>0$ ). Hence, given $\alpha \geq \frac{1}{2}$, we need to find the order of decay of the probability $\mathcal{P}\left\{\left|n(r)-r^{2}\right|>r^{\alpha}\right\}$.

1.4.1. Naïve heuristics. The aforementioned similarity between the zero process $\mathcal{Z}$ and independent complex Gaussian perturbations $\mathcal{S}$ of the lattice $\sqrt{\pi} \mathbb{Z}^{2}$ helps to guess the correct answer.

We fix the parameter $\nu>0$, and consider the random point set $\mathcal{S}_{\nu}=\{\omega+$ $\left.\zeta_{\omega}\right\}_{\omega \in \mathbb{Z}^{2}}$, where $\zeta_{\omega}$ are independent, identical, radially distributed random variables with the tails $\mathcal{P}\left\{\left|\zeta_{\omega}\right|>t\right\}$ decaying as $\exp \left(-t^{\nu}\right)$ as $t \rightarrow \infty$. Set

$$
n_{\nu}(r)=\#\left\{\omega \in \sqrt{\pi} \mathbb{Z}^{2}:\left|\omega+\zeta_{\omega}\right| \leq r\right\} .
$$

Then, for every $\alpha \geq \frac{1}{2}$ and every $\varepsilon>0$,

$$
\exp \left[-r^{\varphi(\alpha, \nu)+\varepsilon}\right]<\mathcal{P}\left\{\left|n_{\nu}(r)-r^{2}\right|>r^{\alpha}\right\}<\exp \left[-r^{\varphi(\alpha, \nu)-\varepsilon}\right],
$$

provided that $r$ is sufficiently big. Here

$$
\varphi(\alpha, \nu)= \begin{cases}2 \alpha-1, & \frac{1}{2} \leq \alpha \leq 1 \\ (\nu+1) \alpha-\nu, & 1 \leq \alpha \leq 2 \\ \left(\frac{1}{2} \nu+1\right) \alpha, & \alpha \geq 2\end{cases}
$$

Actually, one can find much sharper estimates for $\mathcal{P}\left\{\left|n_{\nu}(r)-r^{2}\right|>r^{\alpha}\right\}$.

This suggests that the probability $\mathcal{P}\left\{\left|n(r)-r^{2}\right|>r^{\alpha}\right\}$ we are after should decay as $\exp \left[-r^{\varphi(\alpha)}\right]$ with

$$
\varphi(\alpha)=\varphi(\alpha, 2)= \begin{cases}2 \alpha-1, & \frac{1}{2} \leq \alpha \leq 1 \\ 3 \alpha-2, & 1 \leq \alpha \leq 2 \\ 2 \alpha, & \alpha \geq 2\end{cases}
$$

1.4.2. Jancovici-Lebowitz-Manificat Law. Unfortunately, we do not know how to represent random complex zeroes as independent, or weakly correlated, Gaussian perturbations of the lattice points, so we cannot use the heuristics given above. Nevertheless, we can prove

Theorem 1.3 (JLM Law for random complex zeroes). For every $\alpha \geq \frac{1}{2}$ and every $\varepsilon>0$,

$$
\exp \left[-r^{\varphi(\alpha)+\varepsilon}\right]<\mathcal{P}\left\{\left|n(r)-r^{2}\right|>r^{\alpha}\right\}<\exp \left[-r^{\varphi(\alpha)-\varepsilon}\right]
$$

for all sufficiently large $r>r_{0}(\alpha, \varepsilon)$ with the same $\varphi(\alpha)$ as above.

In [18, Jancovici, Lebowitz and Manificat showed that this law holds for the one-component plasma. Their derivation was not a rigorous one, except for the case of the limiting Ginibre process $\mathcal{G}$. It would be desirable to have a clear explanation why the same Jancovici-Lebowitz-Manificat law holds for the random processes $\mathcal{Z}$, $\mathcal{S}$, and $\mathcal{G}$ in the range $\alpha>1$. 
1.4.3. Comments and questions. The function $\varphi$ from the exponent in the Jancovici-Lebowitz-Manificat Law loses smoothness at three points. Accordingly, there are three different régimes $\left(\frac{1}{2}<\alpha<1,1<\alpha<2\right.$, and $\left.\alpha>2\right)$. The point $\alpha=\frac{1}{2}$ corresponds to the asymptotic normality of $n(r)$, and deviations in the range $\frac{1}{2}<\alpha<1$ are called moderate. In this range, the deviation $\left|n(r)-r^{2}\right|$ is small compared to the length of the circumference $\{|z|=r\}$. In this case, the theorem was proven by Nazarov, Sodin, and Volberg [37. The point $\alpha=1$ corresponds to the classical large deviations principle. In the range $1<\alpha<2$, the deviation is already big compared to the length of the boundary circumference, but is still small compared to the area of the disk $\{|z| \leq r\}$. In this case, the lower bound for $\mathcal{P}\left\{\left|n(r)-r^{2}\right|>r^{\alpha}\right\}$ is due to Krishnapur [22], while the upper bound was proven in [37.

The case $\alpha=2$ contains an estimate for the "hole probability" $\mathcal{P}\{n(r)=0\}$. In this case, the theorem was proved by Sodin and Tsirelson [45, Part III]. A very sharp estimate of the hole probability

$$
\log \mathcal{P}\{n(r)=0\}=-\frac{3 e^{2}}{4} r^{4}+O\left(r^{\frac{18}{5}}\right), \quad r \rightarrow \infty,
$$

was recently obtained by Nishry [38; in [39] he extended this asymptotics to a rather wide class of entire functions represented by Gaussian Taylor series. There are two interesting questions pertaining to the hole probability. We have no idea how to find the asymptotics of the expected number of random complex zeroes in the disk $R \mathbb{D}, R \geq r$, conditioned on the hole $\{n(r)=0\}$. We also do not know how to extend Nishry's result from the unit disk to other bounded domains $G$. It seems plausible that for a large class of bounded domains $G$,

$$
\log \mathcal{P}\{n(r G)=0\}=-(\kappa(G)+o(1)) r^{4}, \quad r \rightarrow \infty,
$$

with $\kappa(G)>0$. If this is true, how does $\kappa(G)$ depend on $G$ ?

The range $\alpha>2$ in the Jancovici-Lebowitz-Manificat Law is the "overcrowding" régime. In [22], Krishnapur proved that for $\alpha>2$,

$$
\log \mathcal{P}\left\{n(r)>r^{\alpha}\right\}=-\left(\frac{1}{2} \alpha-1+o(1)\right) r^{2 \alpha} \log r, \quad r \rightarrow \infty .
$$

The bounds in Theorem 1.3 are not too tight. As we've already mentioned, in some cases, much better bounds are known. It would be good to improve precision of Theorem 1.3 in other cases. For instance, to show that for $\alpha \leq 2$ and for $\delta>0$ there exists the limit

$$
\lim _{r \rightarrow \infty} \frac{\log \mathcal{P}\left\{\left|n(r)-r^{2}\right|>\delta r^{\alpha}\right\}}{r^{\varphi(\alpha)}}
$$

and to find its value.

1.4.4. Moderate deviations for smooth linear statistics. Here is a recent result of Tsirelson [52]: 
Theorem 1.4. Let $h \in C_{0}^{2}$. Then

$$
\log \mathbb{P}\left\{r n(r, h)>t \sigma\|\Delta h\|_{L^{2}}\right\}=(1+o(1)) \log \left(\frac{1}{\sqrt{2 \pi}} \int_{t}^{\infty} e^{-x^{2} / 2} \mathrm{~d} x\right)
$$

and

$$
\log \mathbb{P}\left\{r n(r, h)<-t \sigma\|\Delta h\|_{L^{2}}\right\}=(1+o(1)) \log \left(\frac{1}{\sqrt{2 \pi}} \int_{t}^{\infty} e^{-x^{2} / 2} \mathrm{~d} x\right),
$$

as $r \rightarrow \infty, t>0$, and $t \frac{\log ^{2} r}{r} \rightarrow 0$. Here, $\sigma^{2}=\frac{\zeta(3)}{16 \pi}$ (cf. (1.1) ).

The proof of this theorem is quite intricate. Note that it gives bounds that are much sharper than the ones in Theorem 1.3. In the case $t=$ const, Theorem 1.4 gives another proof of the asymptotic normality of smooth linear statistics of random complex zeroes.

It is not clear whether the assumption $t \frac{\log ^{2} r}{r} \rightarrow 0$ can be replaced by a more natural one $\frac{t}{r} \rightarrow 0$. To the best of our knowledge, until now, there have been no results about large or huge deviations for smooth linear statistics of random complex zeroes when $t$ is comparable or much larger than $r$.

\section{Uniformity of spreading of random complex ze- roes over the plane}

Let $Z$ be a point process in $\mathbb{R}^{d}$ with the distribution invariant with respect to the isometries of $\mathbb{R}^{d}$. A natural way to check how evenly the process $Z$ is spread over $\mathbb{R}^{d}$ is to find out how far the counting measure

$$
n_{Z}=\sum_{a \in Z} \delta_{a}
$$

of the set $Z$ ( $\delta_{a}$ is the unit mass at $a$ ) is from the Lebesgue measure $m_{d}$ in $\mathbb{R}^{d}$. We describe a convenient way to measure the distance between $n_{Z}$ and $m_{d}$.

Suppose that the mean number of points of $Z$ per unit volume equals 1 . We want to partition the whole space $\mathbb{R}^{d}$, except possibly a subset of zero Lebesgue measure, into disjoint sets $B(a)$ of Lebesgue measure 1 indexed by sites $a \in Z$ in such a way that each set $B(a)$ is located not too far from the corresponding site $a \in Z$. In other words, we are looking for a measurable map $T: \mathbb{R}^{d} \rightarrow Z$ such that for each $a \in Z$, we have $m_{d}\left(T^{-1}\{a\}\right)=1$. We also want the distances $|T x-x|$ to be not too large. The map $T$ is called the transportation (a.k.a. "matching", "allocation", "marriage", etc.) of the Lebesgue measure $m_{d}$ to the set $Z$.

Alternatively, we can fix a lattice $\Gamma \subset \mathbb{R}^{d}$ with cells of unit volume, and look for a bijection $\Theta: \Gamma \rightarrow Z$ for which the distances $|\Theta \gamma-\gamma|, \gamma \in \Gamma$, are not too large. Since for each two lattices $\Gamma_{1}, \Gamma_{2}$ with cells of the same volume, there is a bijection $\theta: \Gamma_{1} \rightarrow \Gamma_{2}$ with $\sup \left\{|\theta \gamma-\gamma|: \gamma \in \Gamma_{1}\right\}<\infty$, the choice of the lattice is not important, so we can take $\Gamma=\mathbb{Z}^{d}$. 
Since we deal with random discrete sets $Z$, the corresponding transportation maps $T$ (or the bijections $\Theta$ ) will be random maps. In interesting cases (including the random complex zeroes $\mathcal{Z}$ ), almost surely, the transportation distances $|T x-x|$ are unbounded, so we are interested in the rate of decay of the probability tails $\mathcal{P}\{|T x-x|>R\}$ as $R \rightarrow \infty$.

Here we present two approaches to this problem developed in [45, Part II] and in [36. Though we discuss only the random complex zeroes $\mathcal{Z}$, we believe that both approaches should work for other natural translation invariant point processes. At last, we recall that the random complex zero process $\mathcal{Z}$ has intensity $\pi$, not 1 . For this reason, we will look for a transportation of the measure $\pi m_{2}$ to $\mathcal{Z}$, and for a bijection between the lattice $\sqrt{\pi} \mathbb{Z}^{2}$ and $\mathcal{Z}$.

\subsection{Random complex zeroes as randomly perturbed lattice points.}

Theorem 2.1 (Existence of well-localized bijection). There exists a translation invariant random function $\xi: \mathbb{Z}^{2} \rightarrow \mathbb{C}$ such that

(a) the random set $\left\{\gamma+\xi(\gamma): \gamma \in \sqrt{\pi} \mathbb{Z}^{2}\right\}$ is equidistributed with the random complex zeroes $\mathcal{Z}$;

(b) $\mathcal{P}\{|\xi(0)|>R\} \leq \exp \left(-c R^{4} / \log R\right)$ for some $c>0$ and every $R \geq 2$.

The theorem is almost optimal since the probability that the disk of radius $\lambda \geq 1$ is free of random complex zeroes is not less than $\exp \left(-C \lambda^{4}\right)$. It seems that the question about the existence of a matching between the lattice and $\mathcal{Z}$ with tails decaying as $\exp \left(-c \lambda^{4}\right)$ remains open as well as the same question for the Gaussian perturbations $\mathcal{S}$ of the lattice points and for the limiting Ginibre process $\mathcal{G}$.

It would be interesting to find a version of Theorem 2.1 with weakly correlated perturbations $\xi_{k, l}$ at large distances. This could shed some light to the reasons hidden behind the Jancovici-Lebowitz-Manificat Law.

2.1.1. Uniformly spread sequences in $\mathbb{R}^{d}$. The proof of Theorem 2.1 is based on a deterministic idea which might be useful in study of the uniformity of spreading of sequences and measures. We need to establish the bijection between the sets $\mathcal{Z}$ and $\sqrt{\pi} \mathbb{Z}^{2}$ with controlled tails of the distances $\left|\xi_{k, l}\right|$. First we look at a simpler situation when $\left|\xi_{k, l}\right|$ are uniformly bounded. It is too much to expect from a typical zero set, but let us try anyway. We say that the set $Z \in \mathbb{R}^{d}$ is $r$-uniformly spread over $\mathbb{R}^{d}$ (with density 1 ) if there exists a bijection between $Z$ and a lattice with the unit volume of the cell such that the distances between $Z$ and the corresponding lattice points do not exceed $r$. If such a bijection exists then clearly

$$
n(U) \leq \nu\left(U_{+r}\right) \quad \text { and } \quad \nu(U) \leq n\left(U_{+r}\right)
$$

for every $U \subset \mathbb{R}^{d}$; here $U_{+r}$ stands for the $r$-neighbourhood of $U, n$ is the counting measure of the set $Z$, and $\nu$ is the counting measure of the lattice. In fact, (2.1) is not only necessary but also sufficient, which is basically a well-known locally finite marriage lemma due to M. Hall and R. Rado. When verifying condition (2.1), we 
can replace $\nu$ by the Lebesgue measure $m_{d}$ at the expense of adding a constant to $r$.

Now, given a locally finite measure $\mu$ on $\mathbb{R}^{d}$, we define $\operatorname{Di}(\mu)$ as the infimum of $r \in(0, \infty)$ such that

$$
\mu(X) \leq m_{d}\left(X_{+r}\right) \quad \text { and } \quad m_{d}(X) \leq \mu\left(X_{+r}\right)
$$

for every bounded Borel set $X \subset \mathbb{R}^{d}$. The range of Di is $[0,+\infty]$ with the both ends included. The following theorem gives a useful upper bound for $\operatorname{Di}(\mu)$ in terms of the potential $u$ :

Theorem 2.2 (Upper bound for the transportation distance). Let $u$ be a locally integrable function in $\mathbb{R}^{d}$ such that $\Delta u=\mu-m_{d}$ in the sense of distributions. Then

$$
\operatorname{Di}(\mu) \leq \text { Const }_{d} \cdot \inf _{r>0}\left\{r+\sqrt{\left\|u * \chi_{r}\right\|_{\infty}}\right\} .
$$

Here, $\chi_{r}$ is the indicator function of the ball of radius $r$ centered at the origin normalized by the condition $\left\|\chi_{r}\right\|_{L^{1}}=1$, and $*$ denotes the convolution.

Now, we explain how Theorem 2.1 is deduced from Theorem 2.2. After smoothing, the random potential $U(z)=\log |F(z)|-\frac{1}{2}|z|^{2}$ is locally uniformly bounded. Still, a.s. it remains unbounded in $\mathbb{C}$, so we cannot apply Theorem 2.2 directly. The idea is to introduce on $\mathbb{C}$ a random metric $\rho$ that depends on a G.E.F. F. The metric $\rho$ is small where the random potential $U$ is large. Then we apply a counterpart of Theorem 2.2 with the distances measured in the metric $\rho$, instead of the Euclidean one.

2.1.2. Comments. Theorems 2.1 and 2.2 are taken from Sodin and Tsirelson 45, Part II] (cf. [46). In that paper, the authors proved a weaker subgaussian estimate for the tails, however, after a minor modification of the proof given therein, one gets the result formulated here. Note that the method developed in Sodin and Tsirelson [45, Part II] needs only the existence of a stationary random vector field $v$ in $\mathbb{R}^{d}$ with $\operatorname{div} v=\mu-c_{d} m_{d}$. The tail estimate depends on the rate of decay of the tails of the field $v$ or of the tails of the potential $u$ such that $v=\nabla u$ (if such a $u$ exists).

In the last 20 years, the concept of uniformly spread discrete subsets of $\mathbb{R}^{d}$ has appeared in very different settings. Laczkovich used uniformly spread sets in $\mathbb{R}^{d}$ in his celebrated solution of the Tarski's circle squaring problem 23] (see also 24]). There are various probabilistic counterparts of this notion. For instance, Ajtai, Komlós and Tusnády [1, Leighton and Shor [25], and Talagrand [51] studied a finite counterpart of this, namely, a high probability matching of a system of $N^{2}$ independent random points in the square $[0, N)^{2} \subset \mathbb{R}^{2}$ with the grid $\mathcal{Z}^{2} \cap[0, N)^{2}$.

\subsection{Gradient transportation.}

Unfortunately, the proof of Theorem 2.1 is a pure existence one. It gives us no idea about what the (almost) optimal transportation of the Lebesgue measure to the zero process $\mathcal{Z}$ looks like. Now, we discuss another approach, namely, the 
transportation by the gradient flow of a random potential. The main advantage of this approach is that it yields a quite natural and explicit construction for the desired transportation.

2.2.1. Basins of zeroes. Let $U(z)=\log |F(z)|-\frac{1}{2}|z|^{2}$ be the random potential corresponding to the G.E.F. F. It is easy to check that the distribution of $U$ is invariant with respect to the isometries of the plane. We shall call any integral curve of the differential equation

$$
\frac{d Z}{d t}=-\nabla U(Z)
$$

a gradient curve of the potential $U$. We orient the gradient curves in the direction of decrease of $U$ (this is the reason for our choice of the minus sign in the differential equation above). If $z \notin \mathcal{Z}$, and $\nabla U(z) \neq 0$, by $\Gamma_{z}$ we denote the (unique) gradient curve that passes through the point $z$.

Definition 2.1. Let $a$ be a zero of the G.E.F. F. The basin of $a$ is the set

$$
B(a)=\left\{z \in \mathbb{C} \backslash \mathcal{Z}: \nabla U(z) \neq 0, \text { and } \Gamma_{z} \text { terminates at } a\right\} .
$$

Clearly, each basin $B(a)$ is a connected open set, and $B\left(a^{\prime}\right) \cap B\left(a^{\prime \prime}\right)=\varnothing$ if $a^{\prime}$ and $a^{\prime \prime}$ are two different zeroes of $F$. Remarkably, all bounded basins have the same area $\pi$. Indeed, $\frac{\partial U}{\partial n}=0$ on $\partial B(a)$ and therefore, applying the Green formula and recalling that the distributional Laplacian of $U$ equals $\Delta U=2 \pi \sum_{a \in \mathcal{Z}_{F}} \delta_{a}-2 m$, one gets

$$
1-\frac{m B(a)}{\pi}=\frac{1}{2 \pi} \iint_{B(a)} \Delta U(z) \mathrm{d} m(z)=\frac{1}{2 \pi} \int_{\partial B(a)} \frac{\partial U}{\partial n}(z)|d z|=0 ;
$$

i.e., $m B(a)=\pi$. The picture below helps to visualize what's going on.

\subsubsection{Results.}

Theorem 2.3 (Random partition). Almost surely, each basin is bounded by finitely many smooth gradient curves (and, thereby, has area $\pi$ ), and

$$
\mathbb{C}=\bigcup_{a \in \mathcal{Z}} B(a)
$$

up to a set of measure 0 (more precisely, up to countably many smooth boundary curves).

The tails of this random partition have three characteristic exponents $\mathbf{1}, \frac{\mathbf{8}}{\mathbf{5}}$, and 4. The probability that the diameter of a particular basin is greater than $R$ is exponentially small in $R$. Curiously enough, the probability that a given point $z$ lies at a distance larger than $R$ from the zero of $F$ it is attracted to decays much faster: as $e^{-R^{8 / 5}}$. This is related to long thin tentacles seen on the picture around some basins. They increase the typical diameter of the basins though the 


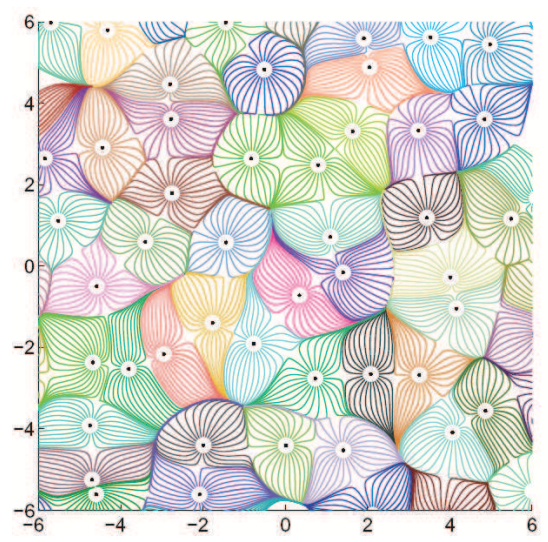

Figure 2. Random partition of the plane into domains of equal area generated by the gradient flow of the random potential $U$ (figure by $\mathrm{M}$. Krishnapur). The lines are gradient curves of $U$, the black dots are random zeroes. Many basins meet at the same local maximum, so that two of them meet tangentially, while the others approach it cuspidally forming long, thin tentacles.

probability that a given point $z$ lies in such a tentacle is very small. At last, given $\varepsilon>0$, the probability that it is impossible to throw away $\varepsilon \%$ of the area of the basin so that the diameter of the remaining part is less than $R$ decays as $e^{-R^{4}}$. All three exponents are optimal. The proofs of these results rely on the following long gradient curve theorem.

Theorem 2.4 (Long gradient curve). Let $R \geq 1$. Let $Q(R)$ be the square centered at the origin with side length $R$. The probability of the event that there exists a gradient curve joining $\partial Q(R)$ with $\partial Q(2 R)$ does not exceed $C e^{-c R(\log R)^{3 / 2}}$.

The proof of this theorem is, unfortunately, rather long and complicated. It might be helpful for the reader to look at the first version of [36] posted in the arxiv where the authors gave a more transparent proof of a weaker upper bound $C e^{-c R \sqrt{\log R}}$ in the long gradient curve theorem.

2.2.3. Comments and questions. Gradient transportation was introduced by Sodin and Tsirelson [45, Part II] and studied by Nazarov, Sodin, Volberg in [36].

There are several questions related to the statistics of our random partition of the plane. It is not difficult to show that, almost surely, any given point $z \in \mathbb{C}$ belongs to some basin. We denote that basin by $B_{z}$, and the corresponding sink by $a_{z}$. We say that two basins are neighbours if they have a common gradient curve on the boundary. By $N_{z}$ we denote the number of basins $B$ neighbouring the basin $B_{z}$. Clearly, $N_{z}$ equals the number of saddle points of the potential $U$ connected with the sink $a_{z}$ by gradient curves. Heuristically, since almost surely 
each saddle point is connected with two sinks,

$$
\mathcal{E} N_{z}=2 \frac{\text { mean number of saddle points per unit area }}{\text { mean number of zeroes per unit area }} .
$$

Douglas, Shiffman and Zelditch proved in [15] that the mean number of saddle points of $U$ per unit area is $\frac{4}{3 \pi}$. (They proved this for another closely related "elliptic model" of Gaussian polynomials. It seems that their proof also works for G.E.F.'s) This suggests that $\mathcal{E} N_{z}=\frac{8}{3}$. Another characteristic of the random partition is the number of basins that meet at the same local maximum. Taking into account the result from [15, we expect that its average equals 8 .

The next question concerns the topology of our random partition of the plane. By the skeleton of the gradient flow we mean the connected planar graph with vertices at local maxima of $U$ and edges corresponding to the boundary curves of the basins. The graph may have multiple edges and loops. We do not know whether there are any non-trivial topological restrictions on finite parts of the skeleton that hold almost surely.

In 11] Chatterjee, Peled, Peres, Romik applied the ideas from [36 to study the gradient transportation of the Lebesgue measure to the Poisson point process in $\mathbb{R}^{d}$ with $d \geq 3$ (they called it 'gravitational allocation'). Their work required a delicate and thorough analysis of the behaviour of the Newtonian potential of the Poisson point process. It's worth mentioning that a very different construction of the stable marriage between the Lebesgue measure $m_{d}$ and the Poisson process in $\mathbb{R}^{d}$ with $d \geq 2$ was developed by Hoffman, Holroyd and Peres in [20. The case $d=2$ is especially interesting: see the recent work by Holroyd, Pemantle, Peres, Schramm [19.

\section{Almost independence and correlations}

\subsection{Almost independence at large distances.}

The covariance function of the normalized Gaussian process $F^{*}(z)=F(z) e^{-\frac{1}{2}|z|^{2}}$ equals

$$
e^{z \bar{w}-\frac{1}{2}|z|^{2}-\frac{1}{2}|w|^{2}}=e^{\mathrm{i} \operatorname{Im}(z \bar{w})-\frac{1}{2}|z-w|^{2}},
$$

which decays very fast as $|z-w|$ grows. This suggests an idea that the zeroes of G.E.F.'s must be "almost independent" on large distances. Still the precise formulation of this independence property is not obvious: due to analyticity of $F$, if we know the process $F^{*}$ in a neighbourhood of some point, we know it everywhere on the plane.

It is not difficult to show that two standard complex Gaussian random variables with small covariance can be represented as small perturbation of two independent standard complex Gaussian random variables. Developing this idea, we show that if $\left\{K_{j}\right\}$ is a collection of well-separated compact sets, then the restrictions $\left.F^{*}\right|_{K_{j}}$ of normalized process $F^{*}$ can be simultaneously approximated by restrictions $\left.F_{j}^{*}\right|_{K_{j}}$ 
of normalized independent realizations of G.E.F.'s $F_{j}$ with high precision and the probability very close to 1 . This is a very useful principle that lies in the core of the proofs of most of the results described above. Here is the precise statement [34]:

Theorem 3.1 (Almost independence). Let $F$ be a G.E.F.. There exists a numerical constant $A>1$ with the following property. Given a family of compact sets $K_{j}$ in $\mathbb{C}$ with diameters $d\left(K_{j}\right)$, let $\lambda_{j} \geq \max \left\{d\left(K_{j}\right), 2\right\}$. Suppose that $A \sqrt{\log \lambda_{j}}$ neighbourhoods of the sets $K_{j}$ are pairwise disjoint. Then

$$
F^{*}=F_{j}^{*}+G_{j}^{*} \quad \text { on } K_{j},
$$

where $F_{j}$ are independent G.E.F.'s and for every $j$, we have

$$
\mathcal{P}\left\{\max _{K_{j}}\left|G_{j}^{*}\right| \geq \lambda_{j}^{-1}\right\} \lesssim e^{-\lambda_{j}} .
$$

Less general versions of this result were proven in [36, 37.

The proof of Theorem 3.1 goes as follows. First, for each compact set $K_{j}$, we choose a sufficiently dense net $Z_{j}$ and consider the bunch $N_{j}=\left\{v_{z}: z \in Z_{j}\right\}$ of unit vectors $v_{z}=F^{*}(z)$ in the Hilbert space of complex Gaussian random variables. Since the compact sets $K_{j}$ are well-separated, the bunches $N_{j}$ are almost orthogonal to each other. Then we slightly perturb the vectors $v_{z}$ without changing the angles between the vectors within each bunch $N_{j}$, making the bunches orthogonal to each other. More accurately, we construct new bunches $\widetilde{N}_{j}=\left\{\widetilde{v}_{z}: z \in Z_{j}\right\}$ so that for $z \in Z_{j}, \zeta \in Z_{k}$,

$$
\left\langle\widetilde{v}_{z}, \widetilde{v}_{\zeta}\right\rangle= \begin{cases}\left\langle v_{z}, v_{\zeta}\right\rangle & \text { for } j=k \\ 0 & \text { for } j \neq k\end{cases}
$$

with good control of the errors $\left\|v_{z}-\widetilde{v}_{z}\right\|$. Then we extend the Gaussian bunches $\left\{\widetilde{v}_{z} e^{\frac{1}{2}|z|^{2}}: z \in Z_{j}\right\}$ to independent G.E.F.'s $F_{j}$. The difference $G_{j}=F-F_{j}$ is a random entire function that is small on the net $Z_{j}$ with probability very close to one. At the last step of the proof, using some simple complex analysis, we show that $G_{j}^{*}$ is small everywhere on $K_{j}$.

\subsection{Uniform estimates of $k$-point functions. Clustering.}

There is yet another way (originated in statistical mechanics) to describe point processes by the properties of their $k$-point correlation functions. Recall that the $k$-point function $\rho=\rho_{k}$ of the zero process $\mathcal{Z}$ is a symmetric function on $\mathbb{C}^{k}$ defined outside of the diagonal subset

$$
\operatorname{Diag}\left(\mathbb{C}^{k}\right)=\left\{\left(z_{1}, \ldots, z_{k}\right): z_{i}=z_{j} \text { for some } i \neq j\right\}
$$

by the formula

$$
\rho\left(z_{1}, \ldots, z_{k}\right)=\lim _{\varepsilon \rightarrow 0} \frac{p_{\varepsilon}\left(z_{1}, \ldots, z_{k}\right)}{\left(\pi \varepsilon^{2}\right)^{k}}
$$


where $p_{\varepsilon}\left(z_{1}, \ldots, z_{k}\right)$ is the probability that each disk $\left\{\left|z-z_{j}\right| \leq \varepsilon\right\}, 1 \leq j \leq k$, contains at least one point of $\mathcal{Z}$. The $k$-point functions describe correlations within $k$-point subsets of the point process. Estimates for the $k$-point functions are crucial for understanding many properties of point processes. The following results taken from [35] provide rather complete quantitative information about the behaviour of the $k$-point functions of random complex zeroes.

The first result treats the local behaviour of $k$-point functions. It appears that for a wide class of non-degenerate Gaussian analytic functions, the $k$-point functions of their zeroes exhibit universal local repulsion when some of the variables $z_{1}, \ldots, z_{k}$ approach each other.

Recall that a Gaussian analytic function $f(z)$ in a plane domain $G \subseteq \mathbb{C}$ is the sum

$$
f(z)=\sum_{n} \zeta_{n} f_{n}(z)
$$

of analytic functions $f_{n}(z)$ such that

$$
\sum_{n}\left|f_{n}(z)\right|^{2}<\infty \quad \text { locally uniformly on } G,
$$

where $\zeta_{n}$ are independent standard complex Gaussian coefficients. By $\rho_{f}=$ $\rho_{f}\left(z_{1}, \ldots, z_{k}\right)$ we denote the $k$-point function of the zero set of the function $f$. It is a symmetric function defined outside the diagonal set $\operatorname{Diag}\left(G^{k}\right)$ as in (3.1).

We skip the technical definition of $d$-degeneracy, which we use in the assumptions of the next theorem, and only mention that Gaussian Taylor series (either infinite, or finite)

$$
f(z)=\sum_{n \geq 0} \zeta_{n} c_{n} z^{n}
$$

are $d$-nondegenerate, provided that $c_{0}, c_{1}, \ldots, c_{d-1} \neq 0$. In particular, the G.E.F. is $d$-nondegenerate for every positive integer $d$.

Theorem 3.2 (Local universality of repulsion). Let $f$ be a $2 k$-nondegenerate Gaussian analytic function in a domain $G$, let $\rho_{f}$ be a $k$-point function of zeroes of $f$, and let $K \subset G$ be a compact set. Then there exists a positive constant $C=C(k, f, K)$ such that, for any configuration of pairwise distinct points $z_{1}, \ldots, z_{k} \in K$,

$$
C^{-1} \prod_{i<j}\left|z_{i}-z_{j}\right|^{2} \leq \rho_{f}\left(z_{1}, \ldots, z_{k}\right) \leq C \prod_{i<j}\left|z_{i}-z_{j}\right|^{2} .
$$

The next result is a clustering property of zeroes of G.E.F.'s. It says that if the variables in $\mathbb{C}^{k}$ can be split into two groups located far from each other, then the function $\rho_{k}$ almost equals the product of the corresponding factors. This property is another manifestation of almost independence of points of the process at large distances. It plays a central rôle in the proof of the asymptotic normality theorem 1.2 for bounded measurable functions.

For a non-empty subset $I=\left\{i_{1}, \ldots, i_{\ell}\right\} \subset\{1,2, \ldots, k\}$, we set $Z_{I}=\left\{z_{i_{1}}, \ldots, z_{i_{\ell}}\right\}$. We denote by

$$
d\left(Z_{I}, Z_{J}\right)=\inf _{i \in I, j \in J}\left|z_{i}-z_{j}\right|
$$


the distance between the configurations $Z_{I}$ and $Z_{J}$.

Theorem 3.3 (Clustering property). For each $k \geq 2$, there exist positive constants $C_{k}$ and $\Delta_{k}$ such that for each configuration $Z$ of size $k$ and each partition of the set of indices $\{1,2, \ldots, k\}$ into two non-empty subsets $I$ and $J$ with $d\left(Z_{I}, Z_{J}\right) \geq 2 \Delta_{k}$, one has

$$
1-\varepsilon \leq \frac{\rho(Z)}{\rho\left(Z_{I}\right) \rho\left(Z_{J}\right)} \leq 1+\varepsilon \quad \text { with } \quad \varepsilon=C_{k} e^{-\frac{1}{2}\left(d\left(Z_{I}, Z_{J}\right)-\Delta_{k}\right)^{2}} .
$$

Combining Theorems 3.2 and 3.3 , and taking into account the translation invariance of the point process $\mathcal{Z}$, we obtain a uniform estimate for $\rho_{k}$ valid in the whole $\mathbb{C}^{k}$ :

Theorem 3.4. For each $k \geq 1$, there exists a positive constant $C_{k}$ such that for each configuration $\left(z_{1}, \ldots, z_{k}\right)$,

$$
C_{k}^{-1} \prod_{i<j} \ell\left(\left|z_{i}-z_{j}\right|\right) \leq \rho\left(z_{1}, \ldots, z_{k}\right) \leq C_{k} \prod_{i<j} \ell\left(\left|z_{i}-z_{j}\right|\right),
$$

where $\ell(t)=\min \left(t^{2}, 1\right)$.

The proofs of Theorems 3.2 and 3.3 start with the classical Kac-Rice-Hammersley formula [21, Chapter 3]:

$$
\rho_{f}\left(z_{1}, \ldots, z_{k}\right)=\int_{\mathbb{C}^{k}}\left|\eta_{1}\right|^{2} \ldots\left|\eta_{k}\right|^{2} \mathcal{D}_{f}\left(\eta^{\prime} ; z_{1}, \ldots, z_{k}\right) \mathrm{d} m\left(\eta_{1}\right) \ldots \mathrm{d} m\left(\eta_{k}\right),
$$

where $\mathcal{D}_{f}\left(\cdot ; z_{1}, \ldots, z_{k}\right)$ is the density of the joint probability distribution of the random variables

$$
f\left(z_{1}\right), f^{\prime}\left(z_{1}\right), \ldots, f\left(z_{k}\right), f^{\prime}\left(z_{k}\right)
$$

and $\eta^{\prime}=\left(0, \eta_{1}, \ldots, 0, \eta_{k}\right)^{\mathrm{T}}$ is a vector in $\mathbb{C}^{2 k}$. Since the random variables (3.4) are complex Gaussian, one can rewrite the right-hand side of (3.3) in a more explicit form

$$
\rho_{f}\left(z_{1}, \ldots, z_{k}\right)=\frac{1}{\pi^{2 k} \operatorname{det} \Gamma_{f}} \int_{\mathbb{C}^{k}}\left|\eta_{1}\right|^{2} \ldots\left|\eta_{k}\right|^{2} e^{-\frac{1}{2}\left\langle\Gamma_{f}^{-1} \eta^{\prime}, \eta^{\prime}\right\rangle} \mathrm{d} m\left(\eta_{1}\right) \ldots \mathrm{d} m\left(\eta_{k}\right),
$$

where $\Gamma_{f}=\Gamma_{f}\left(z_{1}, \ldots, z_{k}\right)$ is the covariance matrix of the random variables (3.4). We consider the linear functionals

$$
L f=\sum_{j=1}^{k}\left[\alpha_{j} f\left(z_{j}\right)+\beta_{j} f^{\prime}\left(z_{j}\right)\right]=\frac{1}{2 \pi \mathrm{i}} \int_{\gamma} f(z) r^{L}(z) \mathrm{d} z,
$$

where

$$
r^{L}(z)=\sum_{j=1}^{k}\left[\frac{\alpha_{j}}{z-z_{j}}+\frac{\beta_{j}}{\left(z-z_{j}\right)^{2}}\right]
$$


and $\gamma \subset K$ is a smooth contour that bounds a domain $G^{\prime} \subset K$ that contains the points $z_{1}, \ldots, z_{k}$. Observe that for every vector $\delta=\left(\alpha_{1}, \beta_{1}, \ldots, \alpha_{k}, \beta_{k}\right)^{\mathrm{T}}$ in $\mathbb{C}^{2 k}$, we have

$$
\left\langle\Gamma_{f} \delta, \delta\right\rangle=\mathcal{E}|L f|^{2} .
$$

This observation allows us to estimate the matrix $\Gamma_{f}^{-1}$, and hence the integral on the right-hand side of (3.5), using some simple tools from the theory of analytic functions of one complex variable.

We note that using another approach to analyzing the right-hand side of (3.5), Bleher, Shiffman, and Zelditch proved in 5 that if the points $z_{i}$ are well separated from each other, i.e.,

$$
\min _{i \neq j}\left|z_{i}-z_{j}\right| \geq \delta>0
$$

then some estimate similar to (3.2) holds with a factor $C(k, \delta)$ instead of $C_{k}$. Unfortunately, in this form the result is difficult to apply. For instance, it does not yield the boundedness of the $k$-point functions on the whole $\mathbb{C}^{k}$, and we could not use it for the proof of Theorem 1.2. On the other hand, the result of Bleher, Shiffman and Zelditch is valid for a wider class of zero point processes.

\section{PART II. RANDOM NODAL LINES}

\section{Gaussian spherical harmonic and Gaussian plane wave}

We introduce two remarkable Gaussian random functions closely related to each other: the Gaussian spherical harmonic on the two-dimensional sphere $\mathbb{S}^{2}$ and its scaling limit, the Gaussian plane wave. The study of random plane waves, and in particular, of their nodal portraits, originated in applied mathematics and goes back to M. S. Longuet-Higgins [27] who computed various statistics of nodal lines for Gaussian random waves in connection with the analysis of ocean waves. One of the reasons for the recent interest in random plane waves is the heuristic principle proposed by M. V. Berry [3] called 'the random wave conjecture'. This principle says that the behaviour of high-energy Laplace eigenfunctions in the case when the corresponding geodesic flow is ergodic (the so called 'highly excited quantum chaotic eigenfunctions') should resemble the behaviour of Gaussian random waves. More generally, one would expect that the random spherical harmonic can serve as a good model for the typical behaviour of high-energy Laplace eigenfunctions on a compact surface endowed with a smooth Riemannian metric.

\subsection{Spherical harmonics.}

The spherical harmonic of degree $n$ is a real-valued eigenfunction of the Laplacian (with the minus sign) on the two-dimensional sphere $\mathbb{S}^{2}$ corresponding to the 
eigenvalue $\lambda_{n}=n(n+1)$. Equivalently, it is a trace of a homogeneous harmonic polynomial in $\mathbb{R}^{3}$ of degree $n$ on the unit sphere. Let $\mathcal{H}_{n}$ be the $2 n+1$-dimensional real Hilbert space of spherical harmonics of degree $n$ equipped with the $L^{2}\left(\mathbb{S}^{2}\right)$ norm. The Gaussian spherical harmonic $f$ is the sum

$$
f_{n}=\sum_{k=-n}^{n} \xi_{k} Y_{k}
$$

where $\xi_{k}$ are independent identically distributed mean zero Gaussian (real) random variables with $\mathcal{E} \xi_{k}^{2}=\frac{1}{2 n+1}$ and $\left\{Y_{k}\right\}$ is an orthonormal basis of $\mathcal{H}_{n}$, so $\mathcal{E}\|f\|_{L^{2}\left(\mathbb{S}^{2}\right)}^{2}=$ 1. As a random function, $f_{n}$ does not depend on the choice of the basis $\left\{Y_{k}\right\}$ in $\mathcal{H}_{n}$. Since the scalar product in the Hilbert space $\mathcal{H}_{n}$ is invariant under rotations of the unit sphere, the distribution of the random spherical harmonic $f_{n}$ is also rotation invariant. The covariance function of the Gaussian spherical harmonic equals

$$
\mathcal{E}\left\{f_{n}(x) f_{n}(y)\right\}=P_{n}(\cos \Theta(x, y))
$$

where $\Theta(x, y)$ is the angle between $x$ and $y$, and $P_{n}$ is the Legendre polynomial of degree $n$ normalized by $P_{n}(1)=1$.

\subsection{Random plane waves.}

Now, we turn to the Gaussian plane wave. Informally speaking, it is the twodimensional Fourier transform of the white noise on the unit circumference $\mathbb{S}^{1} \subset \mathbb{R}^{2}$. More formally, we start with the Hilbert space $L_{\mathrm{sym}}^{2}\left(\mathbb{S}^{1}\right)$ that consists of complex valued $L^{2}$-functions $\varphi$ on $\mathbb{S}^{1}$ satisfying the symmetry condition

$$
\varphi(-\lambda)=\overline{\varphi(\lambda)}, \quad \lambda \in \mathbb{S}^{1},
$$

and consider the Fourier image of this space $\mathcal{H}=\mathcal{F} L_{\text {sym }}^{2}\left(\mathbb{S}^{1}\right)$ with the scalar product inherited from $L_{\text {sym }}^{2}\left(\mathbb{S}^{1}\right)$. The space $\mathcal{H}$ consists of real-analytic functions

$$
\Phi(x)=\int_{\mathbb{S}^{1}} e^{\mathrm{i} x \cdot \lambda} \varphi(\lambda) \mathrm{d} m(\lambda)
$$

( $m$ is the Lebesgue measure on $\mathbb{S}^{1}$ ) satisfying the Helmholtz equation $\Delta \Phi+\Phi=0$. The Gaussian plane wave is the sum of the random series

$$
F=\sum_{k} \eta_{k} \Phi_{k}
$$

where $\eta_{k}$ are standard identically distributed independent (real) Gaussian random variables, and $\left\{\Phi_{k}\right\}$ is an orthonormal basis in $\mathcal{H}$. The series converges almost surely, and its sum is again a real analytic function in $\mathbb{R}^{2}$ satisfying the same Helmholtz equation. This construction does not depend on the choice of the basis $\left\{\Phi_{k}\right\}$, and the distribution of the random function $F$ is invariant with respect to translations and rotations of the plane (since the norm in $\mathcal{H}$ is translation and rotation invariant). 
Applying the Fourier transform to the standard orthonormal basis $\left\{\lambda^{m}\right\}_{m \in \mathbb{Z}}$ in $L^{2}\left(\mathbb{S}^{1}\right)$, we get the functions $\mathrm{i}^{m} J_{m}(r) e^{\mathrm{i} m \theta}$ where $(r, \theta)$ are polar coordinates, and $J_{m}$ is the Bessel function of order $m$. This yields a more explicit formula for the Gaussian plane wave:

$$
F(x)=\operatorname{Re} \sum_{m \in \mathbb{Z}} \zeta_{m} J_{|m|}(r) e^{\mathrm{i} m \theta}, \quad x=(r, \theta),
$$

where $\zeta_{m}$ are independent identically distributed complex Gaussian random variables with $\mathcal{E}\left|\zeta_{m}\right|^{2}=2$.

The covariance function of $F$ (which is the same as the reproducing kernel of the space $\mathcal{H}$ ) is given by the Bessel kernel:

$$
\mathcal{E}\{F(x) F(y)\}=J_{0}(|x-y|) .
$$

It is worth mentioning that there are other constructions of random plane 'monochromatic' waves as random linear combinations ('superpositions') of elementary plane waves $e_{\lambda}(x)=e^{\mathrm{i} \lambda \cdot x}$. For instance, following Oravecz, Rudnick, Wigman [40] and Rudnick, Wigman 43, one can consider 'arithmetic random waves'

$$
h_{N}(x)=\operatorname{Re} \sum_{\nu} \zeta_{\nu} e^{2 \pi \mathrm{i}(\nu \cdot x)}
$$

where $\zeta_{\nu}$ are independent identically distributed complex Gaussian random variables with $\mathcal{E}\left|\zeta_{m}\right|^{2}=2$, and the sum is taken over $\nu \in \mathbb{Z}^{2}$ with $|\nu|^{2}=N$. This model remarkably combines analysis and probability theory with the number theory. Its covariance function

$$
\mathcal{E}\left\{h_{N}(x) h_{N}(y)\right\}=\sum_{\nu} \cos 2 \pi(\nu \cdot(x-y))
$$

has a more erratic behaviour than the covariance functions of the Gaussian spherical harmonic and the Gaussian plane wave.

\subsection{Random plane waves as scaling limits of random spher- ical harmonics.}

The Gaussian plane wave $F$ is a scaling limit of the Gaussian spherical harmonic $f_{n}$ when $n \rightarrow \infty$. This is a very special case of a result of Zelditch [55] pertaining to a wide class of Riemannian smooth surfaces, in particular, to all real-analytic Riemannian surfaces.

Informally, for any fixed $R$, the restrictions of the Gaussian functions $f_{n}$ on spherical disks of radius $R / n$ converge as random processes to the restriction of $F$ on the euclidean disk of radius $R$. More formally, we fix a point $x_{0} \in \mathbb{S}^{2}$, and define the random Gaussian function $F_{n}$ on the tangent plane $T_{x_{0}} \mathbb{S}^{2}$ by

$$
F_{n}(u)=\left(f_{n} \circ \exp _{x_{0}}\right)\left(\frac{u}{n}\right)
$$


where $\exp _{x_{0}}: T_{x_{0}} \mathbb{S}^{2} \rightarrow \mathbb{S}^{2}$ is the exponential map. After this scaling, the covariance equals

$$
\mathcal{E}\left\{F_{n}(u) F_{n}(v)\right\}=P_{n}\left(\cos \Theta\left(\exp _{x_{0}}\left(\frac{u}{n}\right), \exp _{x_{0}}\left(\frac{v}{n}\right)\right)\right)
$$

When $n$ goes to $\infty$, the angle between the points $\exp _{x_{0}}\left(\frac{u}{n}\right)$, and $\exp _{x_{0}}\left(\frac{v}{n}\right)$ on the sphere is equivalent to $|u-v| / n$ (locally uniformly in $u$ and $v$ ). Then by classical Hilb's asymptotics of the Legendre polynomials [50, Theorem 8.21.6], the scaled covariance function $\mathcal{E}\left\{F_{n}(u) F_{n}(v)\right\}$ converges to the Bessel kernel $J_{0}(|u-v|)$ locally uniformly in $u$ and $v$.

\section{Nodal portrait}

In most cases, the basic questions about the asymptotic behaviour of the nodal portrait of the Gaussian spherical harmonic $f_{n}$ as $n \rightarrow \infty$, and their counterparts for the Gaussian plane wave in the 'large area limit' are equivalent to each other. In what follows, we concentrate on spherical harmonic versions which are somewhat easier to formulate.

For the spherical harmonic $g \in \mathcal{H}_{n}$, we denote by $Z(g)=\left\{x \in \mathbb{S}^{2}: g(x)=0\right\}$ its nodal set. The connected components of the complement $\mathbb{S}^{2} \backslash Z(g)$ are called nodal domains of $g$. The following (deterministic) facts are special cases of well-known results valid for Laplace eigenfunctions on smooth Riemannian surfaces:

Theorem 5.1. There is a positive numerical constants $C$ such that for each $g \in$ $\mathcal{H}_{n}$, the nodal set $Z(g)$ is a $C n^{-1}$-net on $\mathbb{S}^{2}$.

Theorem 5.2. There is a positive numerical constant $c>0$ such that for each $g \in \mathcal{H}_{n}$, every nodal domain of $g$ contains a disk of radius $\mathrm{cn}^{-1}$.

Together with Figure 3, this gives a very rough idea of how the nodal portraits of a spherical harmonic of large degree should look.

One can find more information about the geometry and the topology of the nodal portraits of spherical harmonics (and more generally, of high-energy Laplace eigenfunctions on smooth Riemannian surfaces) in the pioneering works of Donnelly and Fefferman 12, 13, 14, as well as in the more recent works of Eremenko, Jackobson, and Nadirashvili [16], Mangoubi [29], and Nazarov, Polterovich and Sodin [31. Still, our understanding of nodal portraits is rather restricted, and, in our opinion, this classical area of analysis is very much underdeveloped.

\subsection{Length of the nodal set.}

The basic characteristics of the nodal set of a spherical harmonic $g$ are its length $L(g)$ and the number $N(g)$ of connected components (which is one less than the number of nodal domains). Useful classical integral formulas for the length due to Poincaré and to Kac and Rice make the length a somewhat easier object for a study. For instance, one can prove 


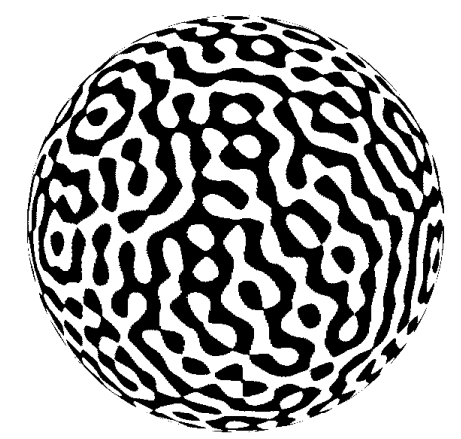

Figure 3. Nodal portrait of the Gaussian spherical harmonic of degree 40 (figure by A. Barnett)

Theorem 5.3. There exists a positive numerical constant $C$ such that for each $g \in \mathcal{H}_{n}, C^{-1} n \leq L(g) \leq C n$

This is a special case of a more general result valid for Laplace eigenfunctions corresponding to large eigenvalues (with $n$ replaced by $\sqrt{\lambda}$ ). The lower bound is valid for any smooth Riemannian surface (this is a result of Brüning [10]), while the upper bound was proven by Donnelly and Fefferman [12] for real-analytic surfaces. In the smooth category, it was conjectured by S. T. Yau, and still remains open in spite of many efforts. Note that one can easily deduce the upper bound in Theorem 5.3 from the fact that spherical harmonics are restrictions of polynomials (that is, without using the deep result of Donnelly and Fefferman).

For the Gaussian spherical harmonic, Bérard showed in 2 that

Theorem 5.4. $\mathcal{E} L\left(f_{n}\right)=\pi \sqrt{2 \lambda_{n}}=\sqrt{2} \pi n+O(1)$

The question about the variance is more delicate. Recently, Wigman [53] confirmed a guess made by M. V. Berry [4] in a slightly different context:

Theorem 5.5. For $n \rightarrow \infty$,

$$
\text { variance of } L\left(f_{n}\right)=\frac{65}{32} \log n+O(1) \text {. }
$$

The proof of this theorem is based on a very careful analysis of asymptotic cancelations that appear in the Kac-Rice integral representation of the variance of $L\left(f_{n}\right)$.

\subsection{The number of connected components.}

There are few classical facts about the number of components $N(g)$. The celebrated Courant nodal domain theorem yields 
Theorem 5.6. For every $g \in \mathcal{H}_{n}, N(g) \leq n^{2}$.

For large $n$, this upper bound was improved by Pleijel [4] to $0.69 n^{2}$. Apparently, the sharp asymptotic upper bound is not known yet. Simple examples show that it cannot be less than $\left(\frac{1}{2}+o(1)\right) n^{2}$. H. Lewy [26] gave an elegant construction of spherical harmonics of any degree $n$ whose nodal sets have one component for odd $n$ and two components for even $n$, which proves that no non-trivial lower bound for $N(g)$ is possible.

Till recently, nothing had been known about the asymptotic properties of the random variable $N\left(f_{n}\right)$ when the degree $n$ is large. The principal difficulty is its non-locality: observing the nodal curves only locally, one cannot make any definite conclusion about the number of connected components. Several years ago Blum, Gnutzmann, and Smilansky [6] raised a question about the distribution of the number of nodal domains of high-energy Laplace eigenfunctions. In the ergodic case, in accordance with Berry's heuristic principle, they suggested to find this distribution for Gaussian random plane waves and performed the corresponding numerics. To compute this distribution, Bogomolny and Schmit proposed in 8 an elegant percolation-like lattice model for description of nodal domains of random Gaussian plane waves. This model completely ignores the (quite big) correlations between the values of the random function $f_{n}$ at different points but nevertheless agrees with numerics pretty well. This agreement is probably due to some hidden 'universality law' rather then the possibility to directly reduce one model to another.

\subsection{Bogomolny-Schmit percolation-like model.}

The Bogomolny-Schmit hypothesis is that the distribution of nodal domains $N\left(f_{n}\right)$ is roughly the same as in the following critical percolation model. Consider the square lattice with the total number of sites equal to $\left(\mathcal{E} L\left(f_{n}\right)\right)^{2}$, that is proportional to $n^{2}$, and change at each site the line crossing to one of the two equiprobable avoided crossing, as shown in the following figure. At different sites, the changes

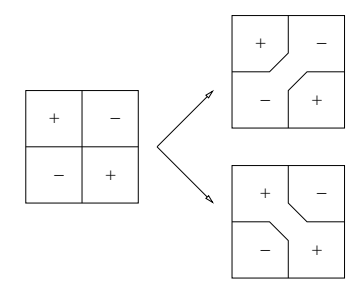

Figure 4. Avoided nodal crossings in the Bogomolny-Schmit model

are independent.

Then Bogomolny and Schmit introduce two dual square lattices: the 'blue one' with vertices at the cells of the grid where the function is positive, and the 'red one' with vertices at the cells of the grid where the function is negative. Each realization of the random process generates two graphs, the blue one whose vertices are the 
blue lattice points and the red one whose vertices are the red lattice points. Two vertices are connected by an edge if the corresponding cells of the grid belong to the same nodal domain of the random function. Each of these graphs uniquely
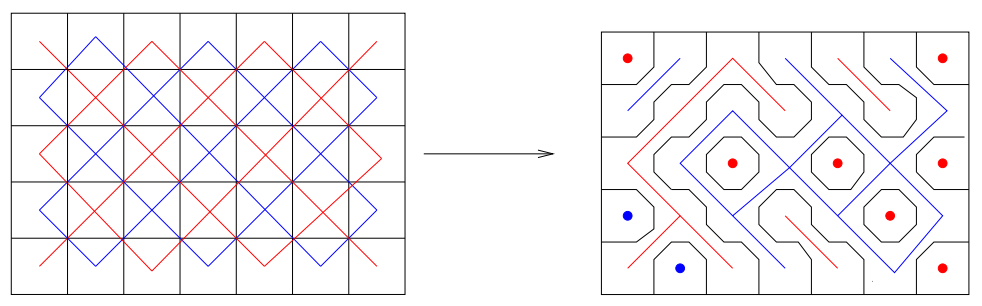

Figure 5. Bond percolation on two dual lattices

determines the whole picture, so it suffices to consider only one of them, and each of them represents the critical bond percolation on the corresponding square lattice. Then using some heuristics coming from statistical mechanics, Bogomolny and Schmit predicted that for $n \rightarrow \infty$,

$$
\mathcal{E} N\left(f_{n}\right)=(a+o(1)) n^{2},
$$

and

$$
\text { variance of } N\left(f_{n}\right)=(b+o(1)) n^{2},
$$

with explicitly computed positive numerical constants $a$ and $b$. They also argued that the fluctuations of the random variable $N\left(f_{n}\right)$ are asymptotically Gaussian when $n \rightarrow \infty$, and concluded their work with a remarkable prediction of the power distribution law for the areas of nodal domains, based on the percolation theory.

It would be interesting to test numerically whether the Bogomolny-Schmit model persists for random linear combinations of plane waves $e^{\mathrm{i} k \cdot x}$ with different wave numbers $k$.

\subsection{Rigorous results.}

Recently, we showed in 32 that, in accordance with one of the Bogomolny and Schmit predictions, $\mathcal{E} N(f) / n^{2}$ tends to a positive limit when $n \rightarrow \infty$, though our proof does not provide us with an explicit value of the limit $a$, so we cannot juxtapose it with the one predicted by Bogomolny and Schmit. In addition, we proved that the random variable $N(f) / n^{2}$ concentrates around this limit exponentially. Since for any spherical harmonic $g \in \mathcal{H}$, the total length of its nodal set $Z(g)$ does not exceed Const $n$, our result yields that, for a typical spherical harmonic, most of its nodal domains have diameters comparable to $1 / n$.

Theorem 5.7 (Number of nodal domains). There exists a constant $a>0$ such that, for every $\varepsilon>0$, we have

$$
\mathcal{P}\left\{\left|\frac{N\left(f_{n}\right)}{n^{2}}-a\right|>\varepsilon\right\} \leq C(\varepsilon) e^{-c(\varepsilon) n}
$$

where $c(\varepsilon)$ and $C(\varepsilon)$ are some positive constants depending on $\varepsilon$ only. 
The exponential decay in $n$ in Theorem 5.7 cannot be improved: we showed that given a positive and arbitrarily small $\kappa$,

$$
\mathcal{P}\left\{N\left(f_{n}\right)<\kappa n^{2}\right\} \geq e^{-C(\kappa) n} .
$$

On the other hand, our proof of Theorem 5.7 gives a very small value $c(\varepsilon) \simeq \varepsilon^{15}$ and it would be nice to reduce the power 15 of $\varepsilon$ to something more reasonable. The question about the variance of $N\left(f_{n}\right)$ remains open.

The last but not least remark is that the proof of Theorem [5.7 uses only relatively simple tools from the classical analysis, which we believe may work in a more general setting of random functions of several real variables (and for higher Betti numbers), while it seems that the Bogomolny-Schmit model is essentially a two-dimensional one.

\subsection{Related work.}

We are aware of several encouraging attempts to tackle similar questions in different contexts. In 49] (motivated by some engineering problems), Swerling estimated from below and from above the mean number of connected components of the level lines $Z(t, f)=\{f=t\}$ of a random Gaussian trigonometric polynomial $f$ of two variables of a given degree $n$. His method is based on estimates of the integral curvature of the level line $Z(t, f)$. The estimates are rather good when the level $t$ is separated from zero, but as $t \rightarrow 0$ they are getting worse and, unfortunately, give nothing when $t=0$.

In the paper 28, Malevich considered $C^{2}$-smooth Gaussian random functions $f$ on $\mathbb{R}^{2}$ with positive covariance function that decays polynomially as the distance between the points tends to infinity. She proved that for $T \geq T_{0}$,

$$
C^{-1} T^{2} \leq \mathcal{E} N(T) \leq C T^{2},
$$

where $N(T)$ is the number of the connected components of the zero set of $f$ that are contained in the square $[0, T] \times[0, T]$, and $C$ is a positive numerical constant. Her proof relies heavily on the positivity property of the covariance function that does not hold for Gaussian spherical harmonics or for Gaussian trigonometric polynomials.

In the recent paper [30, Mischaikow and Wanner studied the following question. Suppose $f$ is a random smooth function on the square $[0,1]^{2}$ with periodic boundary conditions and that the signs of $f$ are computed at the vertices of the grid with mesh $\delta$. How small must $\delta$ be (in terms of the a priori smoothness constants of $f)$ in order to recover the Betti numbers of the sets $\{f>0\}$ and $\{f<0\}$ with probability close to one? In particular, they show that for random trigonometric polynomials of two variables of degree $N$, it suffices to take $\delta=c N^{-2}$ where $c$ is a sufficiently small positive numerical constant. It is possible that their bounds can be significantly improved if instead of recovering the exact values of the Betti numbers one tries to recover them with a small relative error. 


\section{The sketch of the proof of the theorem on the number of nodal domains}

Here, we will describe the main ideas behind the proof of Theorem 5.7, All the details can be found in 32 .

\subsection{The lower bound $\mathcal{E} N\left(f_{n}\right) \geq c n^{2}$.}

This is the simplest part of the story. Denote by $d(.,$.$) the spherical distance.$ Given a point $x \in \mathbb{S}^{2}$ and a large positive constant $C$, we consider the event

$$
\Omega_{x}=\left\{f_{n}(x)>C \text {, and } f_{n}(y)<-C \text { for all } y \text { satisfying } d(x, y)=\frac{\rho}{n}\right\},
$$

where $\rho$ is a constant whose value will be specified below. Clearly, if the event $\Omega_{x}$ occurs, then the disk of radius $\rho / n$ centered at $x$ contains a closed nodal line of $f_{n}$. We claim that

$$
\mathcal{P}\left(\Omega_{x}\right) \geq c>0
$$

where $c$ is a positive constant. The reason is rather straightforward: for every point $x \in \mathbb{S}^{2}$, there exists a function $b_{x} \in \mathcal{H}_{n}$ with $\left\|b_{x}\right\|=1$ such that

$$
b_{x}(x)>c_{0} \sqrt{n} \text { and } b_{x}(y)<-c_{0} \sqrt{n} \text { whenever } d(x, y)=\frac{\rho}{n} .
$$

One can take as $b_{x}$ the zonal spherical harmonic with "pole" $x$. Then we can represent $f_{n}$ in the form

$$
f_{n}=\xi_{0} b_{x}+f_{x}
$$

where $\xi_{0}$ is a Gaussian random variable with $\mathcal{E} \xi_{0}^{2}=\frac{1}{2 n+1}$, and $f_{x}$ is a Gaussian spherical harmonic with $\mathcal{E}\left\|f_{x}\right\|^{2}=\frac{2 n}{2 n+1}$ independent of $\xi_{0}$, and check that with positive probability, the 'perturbation' $f_{x}$ cannot destroy a short nodal curve around point $x$ provided by the function $b_{x}$.

It remains to pack the sphere $\mathbb{S}^{2}$ by $\simeq n^{2}$ disjoint disks of radius $2 \rho / n$. With a positive probability, each of these disks contains a closed nodal line of $f_{n}$. Whence, the lower bound for $\mathcal{E} N\left(f_{n}\right)$.

\subsection{Levy's concentration of measure principle.}

To establish the exponential concentration of the random variable $N\left(f_{n}\right)$ around its median, we would like to use a version of classical Levy's concentration of measure principle.

Given a set $K$, we denote by $K_{+\rho}$ the $\rho$-neighbourhood of $K$. We apply this notation to subsets of $\mathcal{H}_{n}$ and the $L^{2}$-distance, to subsets of $\mathbb{S}^{2}$ and the usual spherical distance, and also to subsets of $\mathbb{R}^{d}$ with the Euclidean distance. The following Gaussian isoperimetric theorem is due to Sudakov and Tsirelson [45] and Borell [7: 
Theorem 6.1. Let $\gamma_{d}$ be the standard Gaussian measure on $\mathbb{R}^{d}$. Let $\Sigma \subset \mathbb{R}^{d}$ be a Borel set, and $\Pi$ be an affine half-space such that

$$
\gamma_{d}(\Sigma)=\gamma_{d}(\Pi) .
$$

Then for each $t>0$,

$$
\gamma_{d}\left(\Sigma_{+\rho}\right) \geq \gamma_{d}\left(\Pi_{+\rho}\right) .
$$

A simple computation shows that if $\gamma_{d}\left(\Pi_{+\rho}\right)$ is not too close to 1 , then $\gamma_{d}(\Pi)$ must be exponentially small in $d$, like $\exp \left[-c \rho^{2} d\right]$. Applying this to the $2 n+1-$ dimensional space $\mathcal{H}_{n}$ of spherical harmonics of degree $n$, we get

Corollary 6.1 (Concentration of Gaussian measure on $\mathcal{H}_{n}$ ). Let $G \subset \mathcal{H}_{n}$ be any measurable set of spherical harmonics. Suppose that the set $G_{+\rho}$ satisfies $\mathcal{P}\left(G_{+\rho}\right)<\frac{3}{4}$. Then $\mathcal{P}(G) \leq 2 e^{-c \rho^{2} n}$.

To use the concentration of measure principle, we need to show that the number $N(f)$ doesn't change too much under slight perturbations of $f$ in the $L^{2}\left(\mathbb{S}^{2}\right)$-norm. Certainly, this is not true for all $f \in \mathcal{H}_{n}$, but we will show that the "unstable" spherical harmonics $f \in \mathcal{H}_{n}$ for which small perturbations can lead to a drastic decrease in the number of nodal lines are exponentially rare. Here is a key lemma which is probably the most novel part of the whole story:

Lemma 6.1 (Uniform lower semi-continuity of $\left.N\left(f_{n}\right) / n^{2}\right)$. For every $\varepsilon>0$, there exist $\rho>0$ and an exceptional set $E \subset \mathcal{H}_{n}$ of probability $\mathcal{P}(E) \leq C(\varepsilon) e^{-c(\varepsilon) n}$ such that for all $f \in \mathcal{H}_{n} \backslash E$ and for all $g \in \mathcal{H}_{n}$ satisfying $\|g\| \leq \rho$, we have

$$
N(f+g) \geq N(f)-\varepsilon n^{2} .
$$

The uniform lower semi-continuity lemma readily yields the exponential concentration of the random variable $N\left(f_{n}\right) / n^{2}$ near its median $a_{n}$. First, consider the set

$$
G=\left\{f \in \mathcal{H}_{n}: N(f)>\left(a_{n}+\varepsilon\right) n^{2}\right\} .
$$

Then for $f \in(G \backslash E)_{+\rho}$, we have $N(f)>a_{n} n^{2}$, and therefore, $\mathcal{P}\left((G \backslash E)_{+\rho}\right) \leq \frac{1}{2}$. Hence, by the concentration of Gaussian measure, $\mathcal{P}(G \backslash E) \leq 2 e^{-c \rho^{2} n}$, and finally,

$$
\mathcal{P}(G) \leq \mathcal{P}(G \backslash E)+\mathcal{P}(E) \leq 2 e^{-c \rho^{2} n}+C(\varepsilon) e^{-c(\varepsilon) n} \leq C(\varepsilon) e^{-c(\varepsilon) n} .
$$

Now, we turn to the set

$$
F=\left\{f \in \mathcal{H}_{n}: N_{f}<\left(a_{n}-\varepsilon\right) n^{2}\right\} .
$$

Then

$$
F_{+\rho} \subset\left\{f \in \mathcal{H}_{n}: N_{f}<a_{n} n^{2}\right\} \cup E,
$$

so that

$$
\mathcal{P}\left(F_{+\rho}\right) \leq \frac{1}{2}+C(\varepsilon) e^{-c(\varepsilon) n}<\frac{3}{4}
$$

for large $n$, and it follows that $\mathcal{P}(F) \leq 2 e^{-c \rho^{2} n}$. 


\subsection{The uniform lower continuity of the functional $f \mapsto N(f)$ outside of an exceptional set.}

Here we explain how we prove Lemma 6.1

6.3.1. Exceptional spherical harmonics $E$ with unstable nodal portraits. Instability of the nodal portrait of a spherical harmonic $f \in \mathcal{H}_{n}$ under small perturbations is caused by points where $f$ and $\nabla f$ are simultaneously small. Let $\alpha$ and $\delta$ be small positive parameters, and let $R$ be a large positive parameter (all of them will depend on $\varepsilon$ from Lemma 6.1). Cover the sphere $\mathbb{S}^{2}$ by $\simeq R^{-2} n^{2}$ disks $D_{j}$ of radius $R / n$ in such a way that the concentric disks $4 D_{j}$ with 4 times larger radius cover the sphere with a bounded multiplicity. We call the disk $D_{j}$ stable if for each $x \in 3 D_{j}$ either $|f(x)| \geq \alpha$ or $|\nabla f(x)| \geq \alpha n$. Otherwise, the disk $D_{j}$ is unstable. We call the spherical harmonic $f \in \mathcal{H}_{n}$ exceptional if the number of unstable disks is at least $\delta n^{2}$, and denote by $E$ the set of all exceptional spherical harmonics of degree $n$.

Lemma 6.2. Given $\delta>0$, there exist positive $C(\delta)$ and $c(\delta)$ such that

$$
\mathcal{P}(E) \leq C(\delta) e^{-c(\delta) n}
$$

provided that the constant $\alpha$ is sufficiently small.

Curiously, the proof of this lemma uses the concentration of measure principle again. It also uses the fact that given $x \in \mathbb{S}^{2}$, the Gaussian random variable $f(x)$ and the Gaussian random vector $\nabla f(x)$ are independent.

6.3.2. Identification of unstable connected components. It remains to show that at most $\varepsilon n^{2}$ nodal components of a stable spherical harmonic can disappear after perturbation of $f$ by another spherical harmonic $g \in \mathcal{H}_{n}$ with sufficiently small $L^{2}$-norm. First, in several steps, we identify possibly 'unstable' connected components of the nodal set $Z(f)$ that can disappear after perturbation, show that their number is small compared to $n^{2}$, and discard them. Then we verify that all other connected components of $Z(f)$ do not disappear after the perturbation.

$\underline{\text { First, }}$ we discard the nodal components $\Gamma$ whose diameter is bigger than $R / n$. By the upper bound in the length estimate in Theorem 5.3, their number is $\lesssim C R^{-1} n^{2}$ which is small compared to $n^{2}$.

With each remaining component $\Gamma$ of the nodal set $Z(f)$ we associate a disk $D_{j}$ such that $D_{j} \cap \Gamma \neq \emptyset$. Then $\Gamma \subset 2 D_{j}$. Since each nodal domain contains a disk of radius $c / n$ (Theorem 5.2 ), the number of components $\Gamma$ intersecting $D_{j}$ (and, thereby, contained in $2 D_{j}$ ) is bounded.

$\underline{\text { Second, }}$ we discard the components $\Gamma$ with unstable disks $D_{j}$. Since $f$ is not exceptional, and since each disk $D_{j}$ cannot intersect too many components contained in $2 D_{j}$, the number of such components is also small compared to $n^{2}$.

$\underline{\text { At last, }}$ we discard the components $\Gamma$ such that

$$
\max _{3 D_{j}}|g| \geq \alpha .
$$


To estimate the number $N$ of such disks, we denote by $D_{j}^{*} \subset 4 D_{j}$ the disk of radius $1 / n$ centered at the point $y_{j}$ where $|g|$ attains its maximum in $3 D_{j}$. By standard elliptic estimates,

$$
\int_{D_{j}^{*}}|g|^{2} \gtrsim n^{-2}\left|g\left(y_{j}\right)\right|=\alpha^{2} n^{-2}
$$

whence

$$
\rho^{2} \geq\|g\|_{L^{2}\left(\mathbb{S}^{2}\right)} \gtrsim N \alpha^{2} n^{-2},
$$

that is, $N \lesssim \rho^{2} \alpha^{-2} n^{2}$. As above, we conclude that the number of components $\Gamma$ affected by this is $\lesssim R^{2} N \lesssim R^{2} \rho^{2} \alpha^{-2} n^{2}$ which is much less than $\varepsilon n^{2}$ provided that $\rho^{2}$ is much less than $\varepsilon \alpha^{2} R^{-2}$.

6.3.3. Verification of stability of the remaining connected components. Now, we claim that the remaining components $\Gamma$ cannot be affected by the perturbation of $f$ by $g$. To see this, we consider the connected component $A_{\Gamma}(t)$ of the set $\{|f|<t\}$ that contains $\Gamma$, and look what may happen with this component when $t$ grows from 0 to $\alpha$.

- As long as $A_{\Gamma}(t)$ stays away from the boundary $\partial\left(3 D_{j}\right)$, it cannot merge with another component of $\{|f|<t\}$ because such a merge can occur only at a critical point of $f$ and there are none of them in $A_{\Gamma}(t) \cap 3 D_{j}$.

- For the same reason, neither of the two boundary curves of $A_{\Gamma}(t)$ can collapse and disappear.

- At last, $A_{\Gamma}(t)$ cannot reach $\partial\left(3 D_{j}\right)$ before it merges with some other component: indeed, if $x \in A_{\Gamma}(t)$ and $A_{\Gamma}(t)$ lies at a positive distance from the boundary $\partial\left(3 D_{j}\right)$ then we can go from $x$ in the direction of $\nabla f$ if $f(x)<0$ and in the direction of $-\nabla f$ if $f(x)>0$. In any case, since $|\nabla f|>\alpha n$ in $A_{\Gamma}(t)$, we shall reach the zero set $Z(f)$ after going the length $1 / n$ or less. Since $\Gamma$ is the only component of $Z(f)$ in $A_{\Gamma}(t)$ before any merges, we conclude that $A_{\Gamma}(t) \subset \Gamma_{+1 / n}$. Recalling that $\operatorname{dist}\left(\Gamma, \partial\left(3 D_{j}\right)\right)>R / n$, we see that, for each $t \leq \alpha, A_{\Gamma}(t)$ stays away from the boundary $\partial\left(3 D_{j}\right)$.

Thus, each component $\Gamma$ lies in a topological annulus $A_{\Gamma}=A_{\Gamma}(\alpha)$ which is contained with its boundary in the open disk $3 D_{j}$ and such that $f=+\alpha$ in one boundary curve of $A_{\Gamma}$ and $f=-\alpha$ on the other. Recalling that $|g|<\alpha$ in $3 D_{j}$, we conclude that $Z(f+g)$ has at least one connected component in $A_{\Gamma}$.

\subsection{Existence of the limit of $\mathcal{E} N\left(f_{n}\right) / n^{2}$.}

We already know that $\mathcal{E} N\left(f_{n}\right) \gtrsim n^{2}$ and that $N\left(f_{n}\right) / n^{2}$ concentrates near its median exponentially. Thus, to finish the proof of Theorem 5.7, it remains to show that the sequence $\left\{\mathcal{E} N\left(f_{n}\right) / n^{2}\right\}$ converges. We deduce this from the fact that the Gaussian spherical harmonic has a scaling limit combined with rotation invariance of the distribution of $f_{n}$. Since this part does not require any new ideas beyond the ones we've already introduced, we just refer the reader to 32] for the details. 


\subsection{Comments and questions.}

Making use of a non-critical version of their percolation model, Bogomolny and Schmit obtained in 9] a series of predictions for the behaviour of the components of level sets which agree with numerics. In a stark contrast, we do not have a rigorous answer even to the following most basic question:

Question 6.1. Prove that for each $\varepsilon>0$ and each $\eta>0$, the probability that the level set $\left\{x \in \mathbb{S}^{2}: f_{n}(x)>\varepsilon\right\}$ has a component of diameter larger than $\eta$ tends to zero as $n \rightarrow \infty$.

One of the reasons for our ignorance is the aforementioned non-locality of the number of connected components. Another essential difficulty is a very slow decay of the correlations which does not allow us to think of restrictions of our process to a collection of well-separated disks as of almost independent processes.

Question 6.2. Estimate the mean number of large components of the nodal set whose diameter is much bigger than $1 / n$. For instance, of those whose diameter is comparable to $n^{-\alpha}$ with $0<\alpha<1$.

Nothing is known about the number of connected components of the nodal set for 'randomly chosen' high-energy Laplace eigenfunction $f_{\lambda}$ on an arbitrary compact surface $M$ without boundary endowed with a smooth Riemannian metric $g$. It is tempting to expect that Theorem 5.7 models what is happening when $M$ is the two-dimensional sphere $\mathbb{S}^{2}$ endowed with a generic Riemannian metric $g$ that is sufficiently close (with several derivatives) to the constant one.

Instead of perturbing the 'round metric' on the sphere $\mathbb{S}^{2}$, one can add a small potential $V$ to the Laplacian on the sphere. The question remains just as hard.

\section{Acknowledgements.}

We are grateful to Manjunath Krishnapur, Yuri Makarychev, Yuval Peres, Leonid Polterovich, Zeév Rudnick, Bernard Shiffman, Boris Tsirelson, Sasha Volberg, and Steve Zelditch for many helpful conversations on the subject of these notes, and to Alex Barnett, Manjunath Krishnapur, and Balint Virág for providing us with inspiring computer generated pictures.

\section{References}

[1] M. Ajtai, J. Komlós and G. Tusnády, On optimal matchings, Combinatorica 4 (1984), 259-264.

[2] P. Bérard, Volume des ensembles nodaux des fonctions propres du laplacien, BonySjöstrand-Meyer seminar, 1984-1985, Exp. No. 14 , 10 pp., École Polytech., Palaiseau, 1985.

[3] M. V. Berry, Regular and irregular semiclassical wavefunctions. J. Phys. A 10 (1977), 2083-2091. 
[4] M. V. Berry, Statistics of nodal lines and points in chaotic quantum billiards: perimeter corrections, fluctuations, curvature, J. Phys. A 35 (2002), 3025-3038.

[5] P. Bleher, B. Shiffman, S. Zelditch, Universality and scaling of correlations between zeros on complex manifolds, Invent. Math. 142 (2000), 351-395. arXiv: math-ph/9904020

[6] G. Blum, S. Gnutzmann, U. Smilansky, Nodal Domains Statistics: A Criterion for Quantum Chaos. Phys. Rev. Letters, 88 (2002), 114101. arXiv:nlin/0109029v1

[7] Chr. Borell, The Brunn-Minkowski inequality in Gauss space. Invent. Math. 30 (1975), 207-216.

[8] E. Bogomolny, C. Schmit, Percolation Model for Nodal Domains of Chaotic Wave Functions. Phys. Rev. Letters, 88 (2002), 114102. arXiv:nlin/0110019v1

[9] E. Bogomolny, C. Schmit, Random wavefunctions and percolation, J. Phys. A 40 (2007), 14033-14043. arXiv:0708.4335 v1

[10] J. Brüning, Über Knoten von Eigenfunktionen des Laplace-Beltrami-Operators, Math. Z. 158 (1978), 15-21.

[11] S. Chatterjee, R. Peled, Y. Peres, D. Romik, Gravitational allocation to Poisson points, Annals of Math., to appear. arXiv:math/0611886 Phase Transitions in Gravitational Allocation, Geom. and Funct. Anal., to appear. arXiv:0903.4647

[12] H. Donnelly, Ch. Fefferman, Nodal sets of eigenfunctions on Riemannian manifolds, Invent. Math. 93 (1988), 161-183.

[13] H. Donnelly, Ch. Fefferman, Nodal sets for eigenfunctions of the Laplacian on surfaces, J. Amer. Math. Soc. bf 3 (1990), 333-353.

[14] H. Donnelly, Ch. Fefferman, Growth and geometry of eigenfunctions of the Laplacian, Analysis and partial differential equations, 635-655, Lecture Notes in Pure and Appl. Math., 122, Dekker, New York, 1990.

[15] M. Douglas, B. Shiffman, and S. Zelditch, Critical points and supersymmetric vacua. I, Comm. Math. Phys. 252 (2004), 325-358. arxiv: math.CV/0402326

[16] A. Eremenko, D. Jakobson, N. Nadirashvili, On nodal sets and nodal domains on $\mathbb{S}^{2}$ and $\mathbb{R}^{2}$, Ann. Inst. Fourier (Grenoble) 57 (2007), 2345-2360. arXiv:math/0611627v2

[17] P. J. Forrester and G. Honner, Exact statistical properties of the zeros of complex random polynomials. J. Phys. A 32 (1999), 2961-2981. arXiv: cond-mat/9811142

[18] B. Jancovici, J. L. Lebowitz, G. Manificat, Large charge fluctuations in classical Coulomb systems. J. Statist. Phys. 72 (1993), 773-787.

[19] A. E. Holroyd, R. Pemantle, Y. Peres, O. Schramm, Poisson Matching, Ann. Inst. Henri Poincaré Probab. Stat. 45 (2009), 266-287. arXiv:0712.1867

[20] C. Hoffman, A. E. Holroyd and Y. Peres, A Stable Marriage of Poisson and Lebesgue, A stable marriage of Poisson and Lebesgue. Ann. Probab. 34 (2006), 1241-1272. arxiv:math.PR/0505668 Tail Bounds for the Stable Marriage of Poisson and Lebesgue, arxiv: math.PR/0507324.

[21] B. Hough, M. Krishnapur, Y. Peres, B. Virág, Zeros of Gaussian Analytic Functions and Determinantal Point Processes, Amer. Math. Soc., 2009. Electronic version available at stat-www. berkeley.edu/ peres/GAF_book.pdf

[22] M. Krishnapur, Overcrowding estimates for zeroes of Planar and Hyperbolic Gaussian analytic functions, J. Statist. Phys. 124 (2006), 1399-1423. arxiv: math.PR/0510588 
[23] M. Laczkovich, Equidecomposability and discrepancy; a solution of Tarski's circlesquaring problem, J. Reine Angew. Math. 404 (1990).

[24] M. Laczkovich, Uniformly spread discrete sets in $\mathbb{R}^{d}$, J. London Math. Soc. (2) 46 (1992).

[25] T. Leighton and P. Shor, Tight bounds for minimax grid matching with applications to the average case analysis of algorithms, Combinatorica 9 (1989), 161-187.

[26] H. Lewy, On the minimum number of domains in which the nodal lines of spherical harmonics divide the sphere. Comm. Partial Differential Equations 2 (1977), 12331244.

[27] M. S. Longuet-Higgins, The statistical analysis of a random, moving surface. Philos. Trans. Roy. Soc. London Ser. A. 249 (1957), 321-387. Statistical properties of an isotropic random surface. Philos. Trans. Roy. Soc. London. Ser. A. 250 (1957), 157174. The statistical geometry of random surfaces. 1962 Proc. Sympos. Appl. Math., Vol. XIII pp. 105-143 American Mathematical Society, Providence, R.I.

[28] T. L. Malevich, Contours that arise whn the zero level is crossed by Gaussian fields. Izv. Acad. Nauk Uzbek. SSR 16 (1972), no 5, 20-23. (Russian)

[29] D. Mangoubi, On the inner radius of a nodal domain, Canad. Math. Bull. 51 (2008), 249-260. arXiv:math/0511329v3 The Volume of a Local Nodal Domain, arXiv:0806.3327 4

[30] K. Mischaikov, T. Wanner, Probabilistic validation of homology computations for nodal domains Ann. Appl. Probab. 17 (2007), 980-1018.

[31] F. Nazarov, L. Polterovich, M. Sodin, Sign and area in nodal geometry of Laplace eigenfunctions, Amer. J. Math. 127 (2005), 879-910. arXiv:math/0402412 v2

[32] F. Nazarov, M. Sodin, On the number of nodal domains of random spherical harmonics. Amer. J. Math. 131 (2009), 1337-1357. arXiv:0706.2409v1

[33] F. Nazarov and M. Sodin, What is a ... Gaussian entire function? Notices Amer. Math. Soc., March, 2010.

[34] F. Nazarov and M. Sodin, Fluctuations in random complex zeroes, In preparation.

[35] F. Nazarov and M. Sodin, Correlation functions for random complex zeroes: strong clustering and weak universality, In preparation.

[36] F. Nazarov, M. Sodin, and A. Volberg, Transportation to random zeroes by the gradient flow, Geom. and Funct. Anal. 17 (2007), 887-935. arXiv:math/0510654

[37] F. Nazarov, M. Sodin, and A. Volberg, The Jancovici - Lebowitz - Manificat law for large fluctuations of random complex zeroes, Commun. Math. Phys. 284 (2008), 833-865. arXiv:0707.3863

[38] A. Nishry, Asymptotics of the Hole Probability for Zeros of Random Entire Functions, Int. Math. Res. Not. IMRN, to appear. arXiv:0903.4970

[39] A. Nishry, The Hole Probability for Gaussian Entire Functions, Israel J. Math., to appear. arXiv:0909.1270

[40] F. Oravecz, Z. Rudnick, I. Wigman, The Leray measure of nodal sets for random eigenfunctions on the torus, Ann. Inst. Fourier (Grenoble) 58 (2008), 299-335. arXiv: math-ph/0609072v2

[41] A. Pleijel, Remarks on Courant's nodal line theorem, Comm. Pure Appl. Math. 9 (1956), 543-550. 
[42] B. Rider and B. Virág, The noise in the circular law and the Gaussian free field, Int. Math. Res. Not. IMRN, no 2 (2007), arXiv:math/0606663 Complex determinantal processes and $H^{1}$ noise, Electron. J. Probab. 12 (2007), 1238-1257, arXiv:math/0608785

[43] Z. Rudnick, I. Wigman, On the volume of nodal sets for eigenfunctions of the Laplacian on the torus, Ann. Henri Poincaré 9 (2008), 109-130. arXiv:math-ph/0702081 v2

[44] M. Sodin, Zeroes of Gaussian analytic functions, European Congress of Mathematics, 445-458, Eur. Math. Soc., Zürich, 2005. arXiv:math/0410343

[45] M. Sodin and B. Tsirelson, Random complex zeroes. I. Asympotic normality, Israel J. Math. 144 (2004), 125-149; II. Perturbed Lattice, ibid 152 (2006), 105-124; III. Decay of the hole probability, ibid 147 (2005), 371-379. arxiv: math.CV/0210090. math.CV/0309449, and math.CV/0312258

[46] M. Sodin and B. Tsirelson, Uniformly spread measures and vector fields, arXiv:0801.2505

[47] A. Soshnikov, Gaussian Limit for Determinantal Random Point Fields, Annals of Probab. 30 (2002), 171-187. arXiv:math/0006037

[48] V. N. Sudakov, B. S. Cirel'son, Extremal properties of half-spaces for spherically invariant measures. Problems in the theory of probability distributions, II. Zap. Naučn. Sem. Leningrad. Otdel. Mat. Inst. Steklov. (LOMI) 41 (1974), 14-24. (Russian)

[49] P. Swerling, Statistical properties of the countours of random surfaces. IRE Trans. Inf. Theory, 8 (1962), 315-321.

[50] G. Szegö, Orthogonal polynomials. Fourth edition. American Mathematical Society, Colloquium Publications, Vol. XXIII. American Mathematical Society, Providence, R.I., 1975.

[51] M. Talagrand, Matching theorems and empirical discrepancy computations using majorizing measures, J. Amer. Math. Soc. 7 (1994), 455-537.

[52] B. Tsirelson, Moderate deviations for random fields and random complex zeroes, arXiv:0801.1050v1

[53] I. Wigman, Fluctuations of the nodal length of random spherical harmonics, arXiv:0907.1648v1

[54] S. Zelditch, From random polynomials to symplectic geometry, XIIIth International Congress on Mathematical Physics (London, 2000), 367-376, Int. Press, Boston, MA, 2001; Asymptotics of polynomials and eigenfunctions, Proceedings of the International Congress of Mathematicians, Vol. II (Beijing, 2002), 733-742, Higher Ed. Press, Beijing, 2002.

[55] S. Zelditch, Real and complex zeros of Riemannian random waves, Spectral analysis in geometry and number theory, 321-342, Contemp. Math., 484, Amer. Math. Soc., Providence, RI, 2009. arXiv:0803.4334

[56] S. Zelditch, Local and global analysis of eigenfunctions, Advanced Lectures in Mathematics (ALM) 7, 545-658 (2008) arXiv:0903.3420v1

F.N.: Mathematics Department, University of Wisconsin-Madison, 480 Lincoln Dr., Madison WI 53706 USA

E-mail: nazarov@math.wisc.edu

M.S.: School of Mathematics, Tel Aviv University, Tel Aviv 69978, Israel

E-mail: sodin@post.tau.ac.il 